\title{
Role of Halogen Interactions in Stabilizing Crystals of [HgX] Based Hybrids
}

\author{
SUMANESH $^{1 *}$, ANJU AWASTHI $^{2}$ and PANKAJ GUPTA ${ }^{3}$ \\ ${ }^{1}$ Research Scholar, Punjab Technical University, Jalandhar, Punjab, India \\ ${ }^{2}$ Department of Chemistry, BCET, Gurdaspur, Punjab, India \\ ${ }^{3}$ Department of Chemistry Govt. Degree College Kathua, J\&K, 184104, India \\ sumneshjasrotia@rediffmail.com
}

Received 31 March 2016 / Accepted 14 April 2016

\begin{abstract}
To analyze the halogen interactions in $\left[\mathrm{HgX}^{-},(\mathrm{X}=\mathrm{Cl}, \mathrm{Br}, \mathrm{I})\right.$, series of inorganic-organic hybrid materials were analyzed through single crystal X-ray crystallographic techniques. It has been observed that few compounds $\mathrm{HgCl}, \mathrm{HgCl} 2, \mathrm{HgCl} 3, \mathrm{HgCl} 6, \mathrm{HgCl}$, $\mathrm{HgCl} 8$ and $\mathrm{HgCl} 9$ of mercuric chloride series validate the phenomenon of halogen interactions with $\mathrm{Cl}-\mathrm{Cl}$ distance less than sum of their van der Waals radii, whereas in mercuric bromide series, this phenomenon is predominant in the compounds of $\mathrm{HgBr} 1, \mathrm{HgBr} 4, \mathrm{HgBr}$, $\mathrm{HgBr}$, $\mathrm{HgBr} 9, \mathrm{HgBr} 10, \mathrm{HgBr} 11, \mathrm{HgBr} 13$ and $\mathrm{HgBr} 16$ with $\mathrm{Br}-\mathrm{Br}$ distance less than sum of their van der Waals radii. Only two compounds in mercuric iodide series HgI1 and HgI15 confirm the phenomenon of halogen interactions whereas these interactions are missing in other remaining compounds of all the three series. The $\mathrm{Hg}-\mathrm{Cl}$ bond distances experiential in the range of 2.346(7) to $2.852(5) \AA$ and $\mathrm{Cl}-\mathrm{Hg}-\mathrm{Cl}$ bond angles in the range of 79.2 to $180^{\circ}$.
\end{abstract}

Keywords: Halogen interactions, Hg motifs, van der Waals radii, Inorganic-Organic hybrid materials, Metal-organic framework

\section{Introduction}

Crystal engineering is concerned with the construction of crystal structures of organic and metal organic species, using design principles that are derived from an understanding of the intermolecular interactions that prevail in molecular solids ${ }^{1}$. Ideally, the crystal to be designed has a specific function whether it is chemical reactivity of a desired kind, an optical, magnetic or electronic property, or still the ability to absorb within the crystal another small molecule ${ }^{2-4}$. To summarise, crystal engineering is the rational design of functional molecular solids. This subject has been in active investigation for around 30 years $^{5-14}$. In the modern context, the concepts of crystal engineering are applicable to any kind of intermolecular assembly whether it is protein ligand recognition, the design of supramolecular polymers, systems for drug delivery or the study of organized media other than crystalline solids. Crystal engineering is accordingly of very wide scope and it has brought together investigators from many disciplines ${ }^{15}$. These include organic chemistry, 
inorganic chemistry, physical chemistry, x-ray crystallography, materials sciences and computational chemistry. The main aim of crystal engineering is to make particular crystal structures from particular molecules.

\section{Inorganic-Organic hybrid materials}

A hybrid of organic and inorganic is a combination of organic materials and inorganic materials ${ }^{16}$ at the molecular level. In such hybrid materials, it is possible to expect very interesting characteristics that are not found in the organic polymer or the inorganic material independently. For example, they can have features such as being flexible like a plastic but have excellent mechanical strength and thermal stability. Hybrid organic-inorganic materials in general represent the natural interface between two worlds of chemistry each with very significant contributions to the field of materials science and each with characteristic properties that result in distinct advantages and limitations ${ }^{17}$.

\section{Halogen $\cdots$ Halogen interactions}

Although crystal engineering is still generally dominated by hydrogen bonding and metalligand interactions, halogen bonds have become increasingly studied and exploited, with a major renaissance over the last ten years. The first use of halogen bonding in liquid crystal formation has been reported by Coronado et al. ${ }^{18}$. Before the discovery of halogen bonding, the approach for crystal engineering involved using halogen bonding, coordination chemistry and inter-ion interactions for the development of liquid-crystalline and solid-crystalline materials. Furthermore, halogen bonding is employed for the organization of radical cationic salts and fabrication of molecular conductors etc. Since the discovery of halogen bonding, new molecular assemblies exist ${ }^{19}$. The range of species capable of adopting halogen bonding interactions indicates a prevalent future for halogen bonds in crystal engineering. As a consequence of the spatially segregated regions of complementary electrostatic potential, interhalogen bonding is also observed with halogen groups acting as both nucelophiles and electrophiles. Desiraju et al., ${ }^{20}$ carried out systematic CSD search on the geometries of these contacts and deduced that the short $\mathrm{X} \cdots \mathrm{X}$ intermolecular distances arise from the specific attractive forces between the two halogen atoms in crystals and the non-spherical shape (polar flattening) of the halogen atoms was the consequence of this interaction. Price et al., ${ }^{21}$ showed that for $\mathrm{X} \cdots \mathrm{X}$ short distances especially chlorine-chlorine interactions could originate from anisotropic effects (non-spherical atomic charge distribution) between elliptically shaped chlorine atoms. The role of $\mathrm{X} \cdots \mathrm{X}$ contacts, appears to be important (irrespective of their cause) in determining supramolecular structures and it may be possible to exploit them directly in crystal engineering and for the design of supramolecular aggregates ${ }^{22}$.

\section{Experimental}

All the structures were pictorially simulated by using Diamond software through ${ }^{23}$ cif-data files and geometry of secondary interactions were analyzed as shown in Table 1-3. IR and Raman spectra tensors were calculated by using fractional co-ordinate obtained from single crystal XRD data as presented in Table 4 . The $\mathrm{HgCl} 1$ crystal structure has been refined up to 0.051 with 1191 reflections and $\mathrm{HgCl} 2$ has $\mathrm{R}$-factor of 0.046 for 3334 reflections. The reliability index of 0.028 has been achieved with 4421 reflections in $\mathrm{HgCl} 3$ whereas its value is 0.026 for 11417 reflections of $\mathrm{HgCl} 4$. The value of $\mathrm{R}$-index is 0.040 for 2904 reflections of $\mathrm{HgCl} 5$ and in $\mathrm{HgCl} 6$ it is 0.034 for 1804 reflections. The well refined crystal structure of $\mathrm{HgCl} 7$ with refined parameter of 0.039 and 0.040 for $\mathrm{HgCl} 8$ shows the structure solution results with 2726 and $3928 \mathrm{~F}>2 \sigma(\mathrm{Fo})$ reflections, respectively. $\mathrm{HgCl} 9$ and $\mathrm{HgCl} 10$ derivatives of the selected series have been refined up to 0.0529 and 0.0322 values, respectively. 
Table 1. Crystal structure data for $[\mathrm{HgX}]^{-},(\mathrm{X}=\mathrm{Cl}, \mathrm{Br}, \mathrm{I})$ compounds

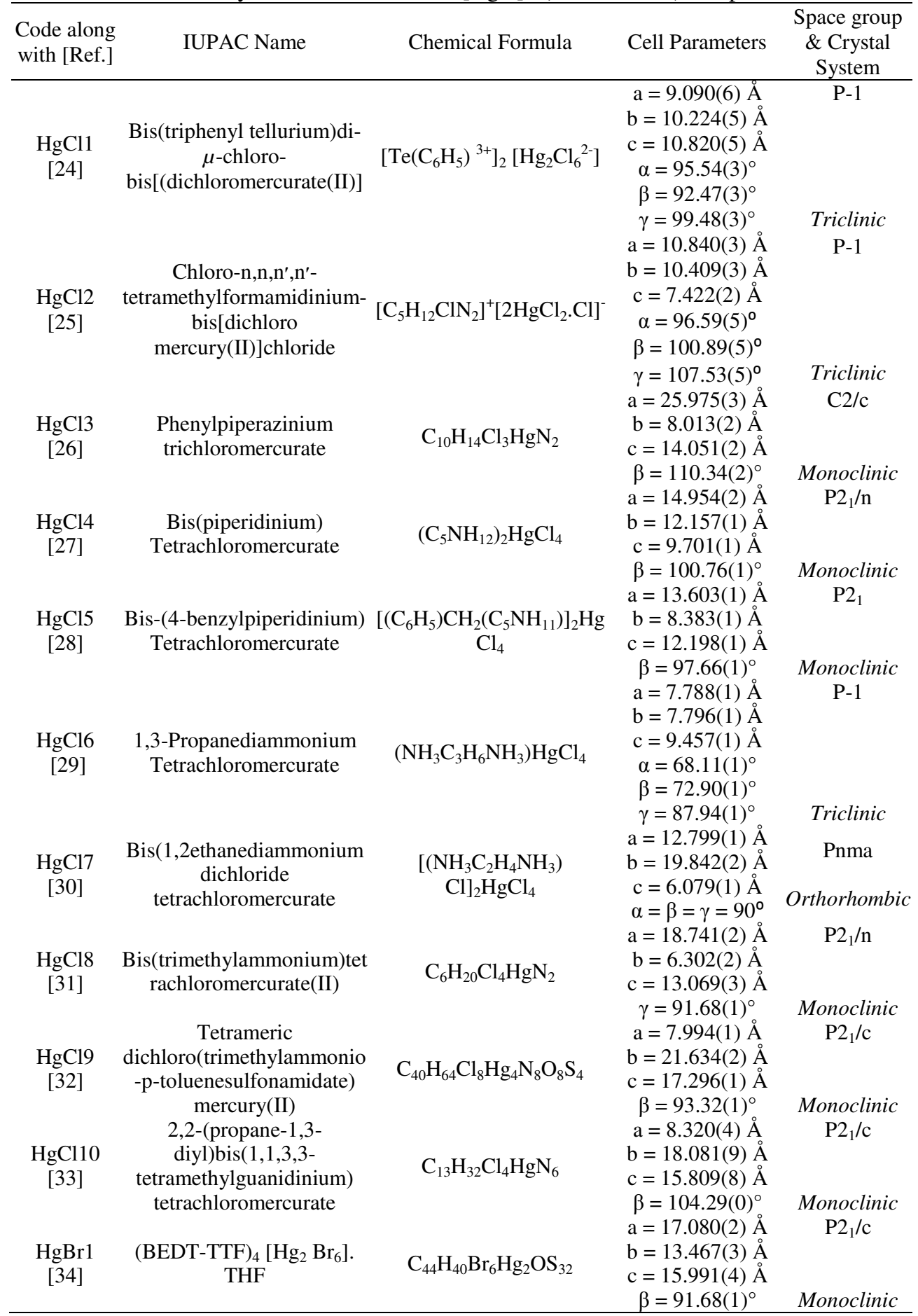




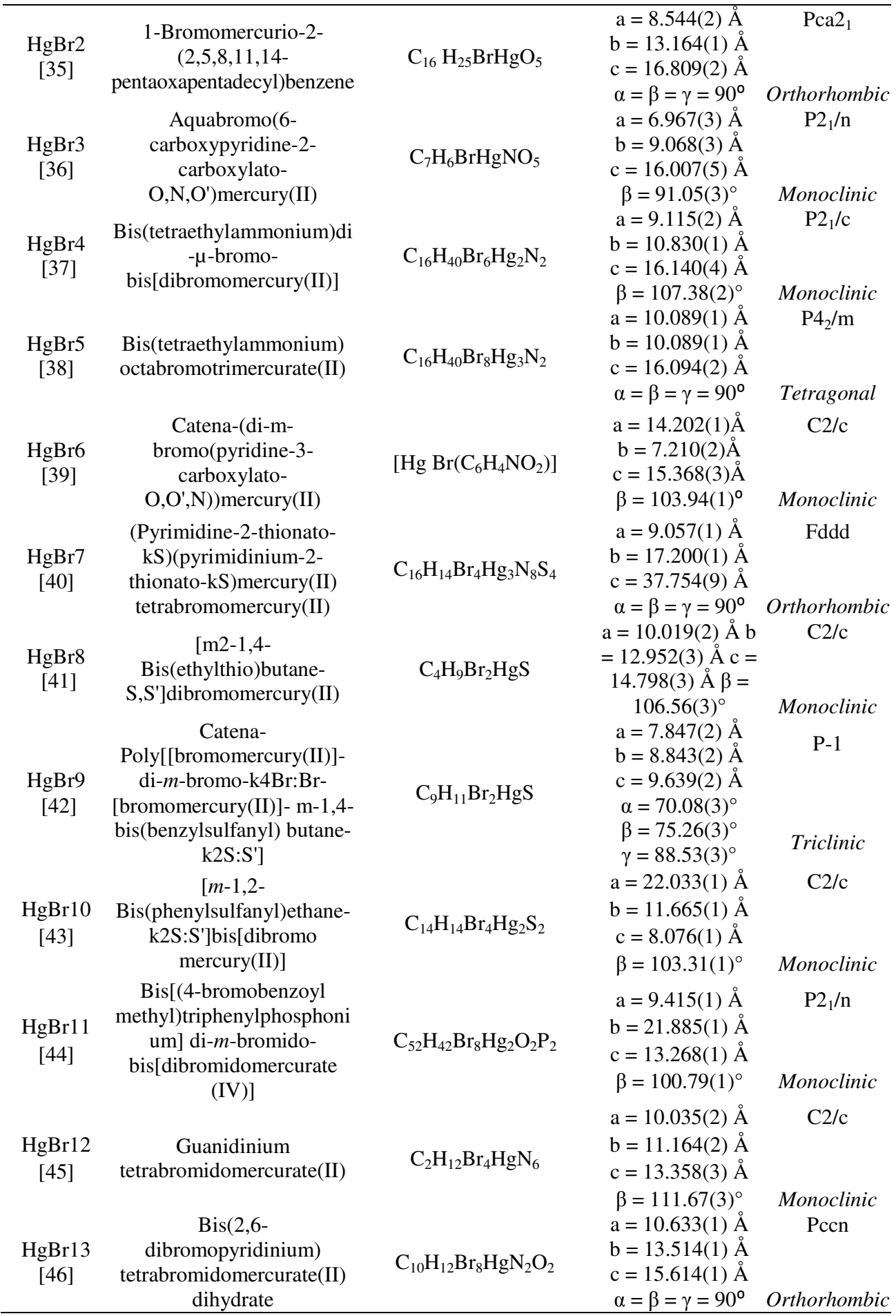




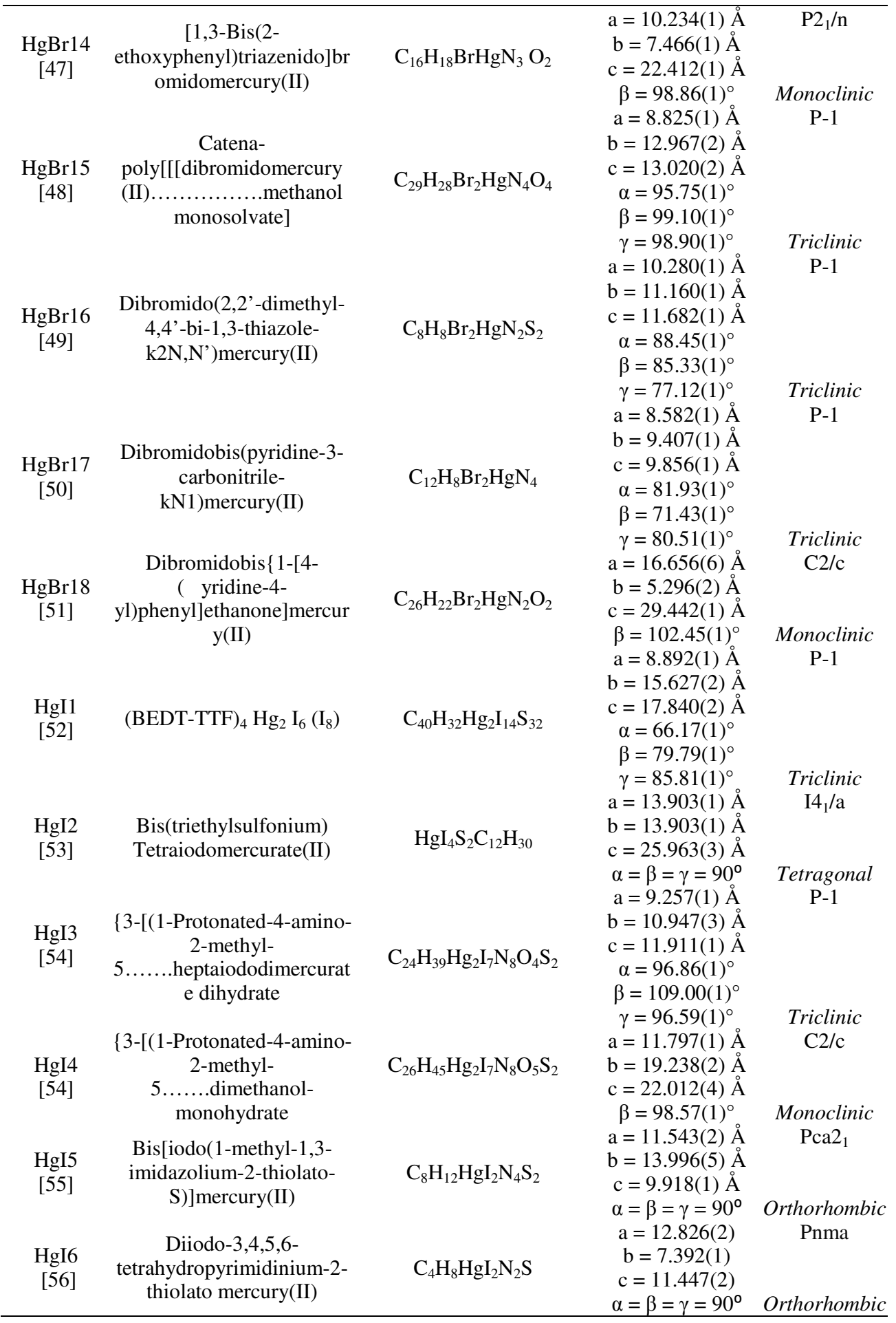




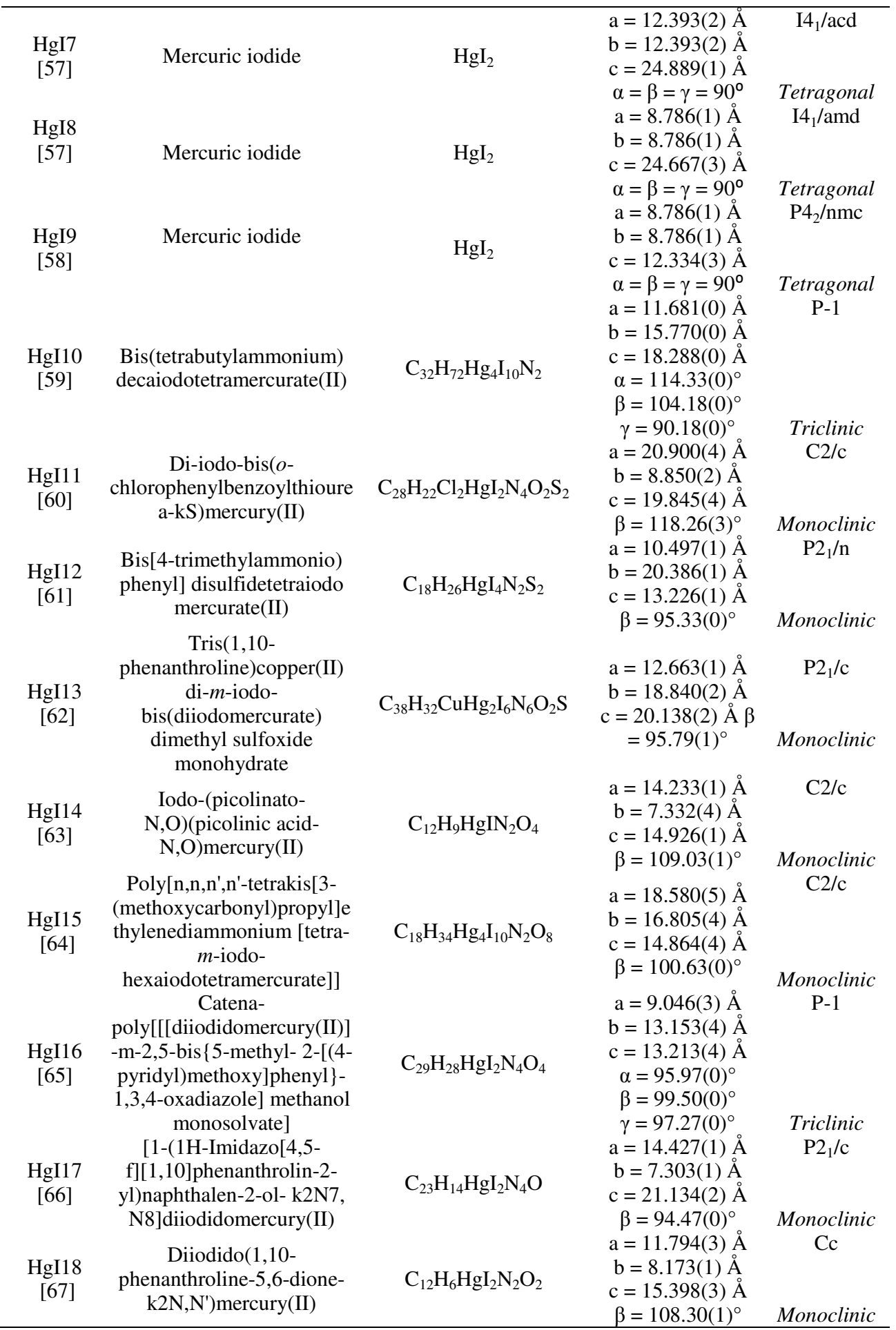


The $\mathrm{HgBr} 1$ crystal structure has been refined up to 0.049 . The value of $\mathrm{R}$ - factor for $\mathrm{HgBr} 2$ and $\mathrm{HgBr} 3$ has been observed to be 0.033 and 0.060 with 4332 and 2658 reflections respectively. The reliability index of 0.032 has been achieved with 2650 reflections in $\mathrm{HgBr} 4$ whereas its value is 0.054 for 2115 reflections in $\mathrm{HgBr} 5$. The value of $\mathrm{R}$ - index is 0.034 for 1804 reflections in $\mathrm{HgBr} 6$ and in $\mathrm{HgBr} 7$ it is 0.043 for 2759 independent reflections. The well refined crystal structure of $\mathrm{Hg} 8$ with refined parameter of 0.042 and 0.032 for $\mathrm{HgBr} 9$ shows the structure solution results with 2737 and 6386 reflections, respectively. $\mathrm{HgBr} 10$ and $\mathrm{HgBr} 11$ derivatives of the selected series have been refined up to 0.029 and 0.032 values for 1361 and 1921 reflections. The cell measurements reflection value of 4420 and 6912 has been used to obtain the refine parameter of 0.053 and 0.026 for the compounds of $\mathrm{HgBr} 12$ and $\mathrm{HgBr} 13$, respectively. The MoK $\alpha$ type of radiations having wavelength 0.71073 have been used to obtain the refine parameter for all the compounds of the selected series ( $\mathrm{HgBr} 1-\mathrm{HgBr} 18)$. The value of $\mathrm{R}$-index is 0.030 for 3967 reflections in $\mathrm{HgBr} 14$ and in $\mathrm{HgBr} 15$ it is 0.104 for 4420 independent reflections. Similarly $\mathrm{HgBr} 16$ crystal structure has been refined up to 0.035 with 6912 reflections and $\mathrm{HgBr} 17$ has the Rfactor of 0.033 for 3967 independent reflections. The cell measurement reflections of 1208 are used to refine the crystal structure of $\mathrm{HgBr} 18$ up to 0.042 with 2358 reflections.

The HgI1 crystal structure has been refined up to 0.070 with 3378 reflections. The value of R-factor for $\mathrm{HgI} 2$ and $\mathrm{HgI} 3$ has been observed to be 0.037 and 0.101 with 1351 and 3912 reflections respectively. The reliability index of 0.064 has been achieved with 4575 reflections in $\mathrm{HgI} 4$ whereas its value is 0.029 for 1428 reflections in $\mathrm{HgI5}$. The value of $\mathrm{R}$ - index is 0.064 for 685 reflections in $\mathrm{HgI} 6$ and in $\mathrm{HgI7}$ it is 0.065 for 585 independent reflections. The well refined crystal structure of $\mathrm{HgI} 8$ with refined parameter of 0.096 and 0.054 for $\mathrm{HgI} 9$ shows the structure solution results with 11615 and 3734 reflections respectively. HgI10 and HgI11 derivatives of the selected series have been refined up to 0.048 and 0.085 values. The cell measurements reflection value of 8558 and 250 have been used to obtain the refine parameter of 0.021 and 0.094 for the compounds of $\mathrm{HgI12}$ and $\mathrm{HgI} 13$ with refine number of reflections of 2149 and 4237 respectively. The value of R-index is 0.087 for 4420 reflections in HgI14 and in HgI15 it is 0.049 for 4351 independent reflections. Similarly, HgI16 crystal structure has been refined up to 0.022 with 3209 independent reflections.

\section{Results and Discussion}

\section{Halogen-Halogen Interactions}

\section{[HgCl] Hybrid materials}

The bond lengths of $\mathrm{Hg}-\mathrm{Cl}$ bond lies in an average range of $2.472 \AA$ to $2.676 \AA$. The graphical projection of bond distances for these derivatives illustrate that most of the data points lie in between 2.45 to $2.55 \AA$ whereas the data points for $\mathrm{HgCl} 2, \mathrm{HgCl} 6$ and $\mathrm{HgCl} 9$ shows the deviation from this linearity as presented in Figure 1. The $\mathrm{Cl}-\mathrm{Hg}-\mathrm{Cl}$ bond angles have wide range from minimum value of 99.30 to $114.90^{\circ}$ for molecule $\mathrm{HgCl} 4$ and maximum range of 88.40 to $180^{\circ}$ for molecule $\mathrm{HgCl} 6$ as shown in Table 2 .

The Graphical view for $\mathrm{Cl} \ldots \mathrm{Cl}$ bond distances illustrates that most of the data points exist in the range of $3.807 \AA$ to $3.949 \AA$ which corroborates that the crystal structures are stabilized by secondary interactions as this range is comparable with van der Waals radii as shown in Figure 2. The minimum value of torsion angle $[\mathrm{Cl}-\mathrm{Hg} \ldots \mathrm{Hg}-\mathrm{Cl}]$ is $4.12(1)^{\circ}$ for the compound $\mathrm{HgCl} 6$ having $\mathrm{x}, \mathrm{y}, 1+\mathrm{z}$ symmetry positions, while its maximum value is $76.02(4)$ for $\mathrm{HgCl} 7$ as shown in Table 3. 


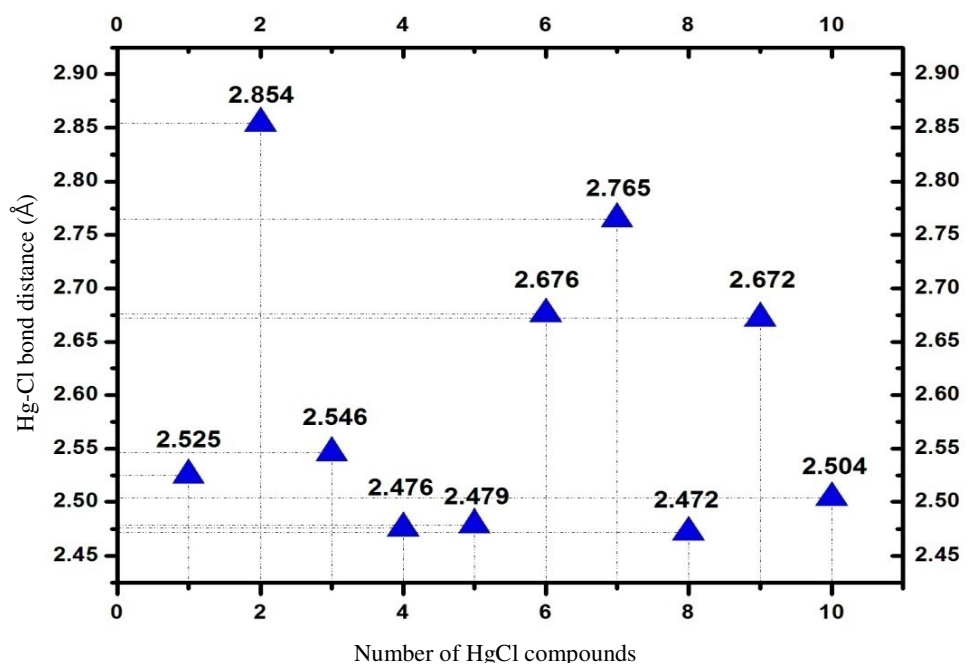

Figure 1. Scatter Plot of the average values of $\mathrm{Hg}-\mathrm{Cl}$ bond distances

Table 2. Crystallographic data for Hg-centered bond distances $(\AA)$ and range of bond angles $\left({ }^{\circ}\right)$ in $[\mathrm{HgX}]^{-},(\mathrm{X}=\mathrm{Cl}, \mathrm{Br}, \mathrm{I})$

\begin{tabular}{|c|c|c|c|c|c|}
\hline Code & $\begin{array}{l}\text { Hg-X bond } \\
\text { distance }(\AA)\end{array}$ & $\begin{array}{l}\mathrm{X}-\mathrm{Hg}-\mathrm{X}\left({ }^{\circ}\right) \\
\text { range }\end{array}$ & Code & $\begin{array}{l}\text { Hg-X bond } \\
\text { distance }(\AA)\end{array}$ & $\begin{array}{l}\mathrm{X}-\mathrm{Hg}-\mathrm{X}\left({ }^{\circ}\right) \\
\text { range }\end{array}$ \\
\hline $\mathrm{HgCl} 1$ & $\begin{array}{l}\mathrm{Hg}-\mathrm{Cl}(1)=2.350(2) \\
\mathrm{Hg}-\mathrm{Cl}(3)=2.350(2) \\
\mathrm{Hg}-\mathrm{Cl}(2)=2.684(2) \\
\mathrm{Hg}-\mathrm{Cl}\left(2^{\prime}\right)=2.717(2)\end{array}$ & $\begin{array}{l}89.60- \\
141.30\end{array}$ & $\mathrm{HgBr} 14$ & $\mathrm{Hg} 1-\mathrm{Br} 1=2.401(1)$ & - \\
\hline $\mathrm{HgCl} 2$ & $\begin{array}{l}\mathrm{Hg}-\mathrm{Cl}(1)=2.312(3) \\
\mathrm{Hg}-\mathrm{Cl}(2)=2.331(2) \\
\mathrm{Hg}-\mathrm{Cl}(3)=2.926(3) \\
\mathrm{Hg}-\mathrm{Cl}(4)=2.983(3) \\
\mathrm{Hg}-\mathrm{Cl}(5)=3.278(3) \\
\mathrm{Hg}-\mathrm{Cl}(6)=3.296(3)\end{array}$ & $79.20-168.50$ & $\mathrm{HgBr} 15$ & $\begin{array}{l}\mathrm{Hg} 1-\mathrm{Br} 2=2.484(1) \\
\mathrm{Hg} 1-\mathrm{Br} 1=2.491(1)\end{array}$ & 145.07 \\
\hline $\mathrm{HgCl} 3$ & $\begin{array}{l}\mathrm{Hg}-\mathrm{Cl} 3=2.346(1) \\
\mathrm{Hg}-\mathrm{Cl} 2=2.365(1) \\
\mathrm{Hg}-\mathrm{Cl} 1=2.624(1) \\
\mathrm{Hg}-\mathrm{Cl} 1=2.852(1)\end{array}$ & $90.90-148.50$ & $\mathrm{HgBr} 16$ & $\begin{array}{l}\mathrm{Hg} 2-\mathrm{Br} 4=2.496(1) \\
\mathrm{Hg} 1-\mathrm{Br} 1=2.497(1) \\
\mathrm{Hg} 2-\mathrm{Br} 3=2.500(1) \\
\mathrm{Hg} 1-\mathrm{Br} 2=2.521(1)\end{array}$ & $126.49-132.85$ \\
\hline $\mathrm{HgCl} 4$ & $\begin{array}{l}\mathrm{Hg}-\mathrm{Cl} 3=2.431(3) \\
\mathrm{Hg}-\mathrm{Cl} 1=2.515(2) \\
\mathrm{Hg}-\mathrm{Cl} 2=2.485(2) \\
\mathrm{Hg}-\mathrm{Cl} 4=2.475(3)\end{array}$ & $99.30-114.90$ & $\mathrm{HgBr} 17$ & $\begin{array}{l}\mathrm{Hg} 1-\mathrm{Br} 1=2.458(1) \\
\mathrm{Hg} 1-\mathrm{Br} 2=2.474(1)\end{array}$ & 159.99 \\
\hline $\mathrm{HgCl} 5$ & $\begin{array}{l}\mathrm{Hg}-\mathrm{Cl} 3=2.424(3) \\
\mathrm{Hg}-\mathrm{Cl} 2=2.430(3) \\
\mathrm{Hg}-\mathrm{Cl} 4=2.450(3) \\
\mathrm{Hg}-\mathrm{Cl}=2.614(2)\end{array}$ & $99.20-115.80$ & $\mathrm{HgBr} 18$ & $\mathrm{Hg} 1-\mathrm{Br} 1=2.470(1)$ & 147.81 \\
\hline $\mathrm{HgCl} 6$ & $\begin{array}{l}\mathrm{Hg} 1-\mathrm{Cl} 4=2.397(2) \\
\mathrm{Hg} 1-\mathrm{Cl} 2=2.821(2) \\
\mathrm{Hg} 1-\mathrm{Cl} 1=2.839(2) \\
\mathrm{Hg} 2-\mathrm{Cl} 3=2.528(2) \\
\mathrm{Hg} 2-\mathrm{Cl} 2=2.701(2) \\
\mathrm{Hg} 2-\mathrm{Cl} 1=2.775(2)\end{array}$ & $88.40-180.00$ & HgI1 & $\begin{array}{l}\mathrm{Hg} 1-\mathrm{I} 1=2.669(3) \\
\mathrm{Hg} 1-\mathrm{I} 3=2.674(3) \\
\mathrm{Hg} 1-\mathrm{I} 2=2.845(3)\end{array}$ & $111.17-127.23$ \\
\hline
\end{tabular}




\begin{tabular}{|c|c|c|c|c|c|}
\hline $\mathrm{HgCl} 7$ & $\begin{array}{l}\mathrm{Hg} 1-\mathrm{Cl} 3=2.378(1) \\
\mathrm{Hg} 1-\mathrm{Cl} 1=2.581(2) \\
\mathrm{Hg} 1-\mathrm{Cl} 2=2.860(3) \\
\mathrm{Hg} 1-\mathrm{Cl} 2=3.243(3)\end{array}$ & $84.69-169.81$ & $\mathrm{HgI} 2$ & $\begin{array}{l}\mathrm{Hg} 1-\mathrm{I} 1=2.780(1) \\
\mathrm{Hg} 2-\mathrm{I} 2=2.790(1)\end{array}$ & $107.74-112.98$ \\
\hline $\mathrm{HgCl} 8$ & $\begin{array}{l}\mathrm{Hg}-\mathrm{Cl} 4=2.414(4) \\
\mathrm{Hg}-\mathrm{Cl} 2=2.442(1) \\
\mathrm{Hg}-\mathrm{Cl} 3=2.430(1) \\
\mathrm{Hg}-\mathrm{Cl} 1=2.604(4)\end{array}$ & $\begin{array}{l}101.40- \\
117.50\end{array}$ & $\mathrm{HgI3}$ & $\begin{array}{l}\mathrm{Hg}-\mathrm{I} 3=2.681(2) \\
\mathrm{Hg}-\mathrm{I} 4=2.736(1) \\
\mathrm{Hg}-\mathrm{I} 2=2.751(5) \\
\mathrm{Hg}-\mathrm{I} 2=2.840(2) \\
\mathrm{Hg}-\mathrm{I} 1=2.971(1)\end{array}$ & $97.90-122.90$ \\
\hline $\mathrm{HgCl} 9$ & $\begin{array}{l}\mathrm{Hg} 2-\mathrm{Cl} 21=2.325(1) \\
\mathrm{Hg} 1-\mathrm{Cl} 11=2.332(1) \\
\mathrm{Hg} 2-\mathrm{Cl} 22=2.334(1) \\
\mathrm{Hg} 1-\mathrm{Cl} 12=2.340(1) \\
\mathrm{Hg} 1-\mathrm{Cl} 11=3.089(1) \\
\mathrm{Hg} 2-\mathrm{Cl} 12=3.065(1) \\
\mathrm{Hg} 1-\mathrm{Cl} 22=3.216(1)\end{array}$ & $84.30-167.60$ & HgI4 & $\begin{array}{l}\mathrm{Hg}-\mathrm{I} 3=2.696(1) \\
\mathrm{Hg}-\mathrm{I} 2=2.779(1) \\
\mathrm{Hg}-\mathrm{I} 4=2.785(1) \\
\mathrm{Hg}-\mathrm{I} 1=2.939(1)\end{array}$ & $98.15-121.62$ \\
\hline $\mathrm{HgCl10}$ & $\begin{array}{l}\mathrm{Hg}-\mathrm{Cl} 3=2.456(1) \\
\mathrm{Hg}-\mathrm{Cl} 2=2.502(1) \\
\mathrm{Hg}-\mathrm{Cl} 1=2.523(1) \\
\mathrm{Hg}-\mathrm{Cl} 4=2.533(1)\end{array}$ & $\begin{array}{l}104.62- \\
120.58\end{array}$ & HgI5 & $\begin{array}{l}\mathrm{Hg}-\mathrm{I} 1=2.781(1) \\
\mathrm{Hg}-\mathrm{I} 2=2.800(1)\end{array}$ & 111.63 \\
\hline $\mathrm{HgBr} 1$ & $\begin{array}{l}\mathrm{Hg}-\mathrm{Br} 3=2.491(1) \\
\mathrm{Hg}-\mathrm{Br} 2=2.494(1) \\
\mathrm{Hg}-\mathrm{Br} 1=2.758(1) \\
\mathrm{Hg}-\mathrm{Br} 1=2.787(1)\end{array}$ & $88.59-132.88$ & HgI6 & $\begin{array}{l}\mathrm{Hg}-\mathrm{I} 1=2.687(1) \\
\mathrm{Hg}-\mathrm{I} 2=2.704(1)\end{array}$ & 123.06 \\
\hline $\mathrm{HgBr} 2$ & $\mathrm{Hg}-\mathrm{Br}=2.443(1)$ & - & $\mathrm{HgI7}$ & $\begin{array}{l}\mathrm{Hg}-\mathrm{I} 2=2.779(3) \\
\mathrm{Hg}-\mathrm{I} 3=2.789(1) \\
\mathrm{Hg}-\mathrm{I} 1=2.795(1)\end{array}$ & $103.22-112.77$ \\
\hline $\mathrm{HgBr} 3$ & $\mathrm{Hg}-\mathrm{Br}=2.412(1)$ & - & HgI8 & $\begin{array}{l}\mathrm{Hg}-\mathrm{I} 3=2.778(2) \\
\mathrm{Hg}-\mathrm{I} 2=2.793(2) \\
\mathrm{Hg}-\mathrm{I} 1=2.806(2)\end{array}$ & $103.76-112.56$ \\
\hline $\mathrm{HgBr} 4$ & $\begin{array}{l}\mathrm{Hg} 1-\mathrm{Br} 2=2.508(1) \\
\mathrm{Hg} 1-\mathrm{Br} 1=2.532(1) \\
\mathrm{Hg} 1-\mathrm{Br} 3=2.734(1) \\
\mathrm{Hg} 1-\mathrm{Br} 1=2.736(1) \\
\mathrm{Hg} 1-\mathrm{Br} 3=2.762(1)\end{array}$ & $90.82-122.15$ & $\mathrm{HgI9}$ & $\begin{array}{l}\mathrm{Hg}-\mathrm{I} 3=2.785(3) \\
\mathrm{Hg}-\mathrm{I} 2=2.788(2) \\
\mathrm{Hg}-\mathrm{I} 1=2.801(3)\end{array}$ & $103.71-112.50$ \\
\hline $\mathrm{HgBr} 5$ & $\begin{array}{l}\mathrm{Hg} 2-\mathrm{Br} 4=2.388(1) \\
\mathrm{Hg} 1-\mathrm{Br} 2=2.517(2) \\
\mathrm{Hg} 1-\mathrm{Br} 3=2.533(2) \\
\mathrm{Hg} 1-\mathrm{Br} 1=2.755(1)\end{array}$ & $92.84-180$ & HgI10 & $\begin{array}{l}\mathrm{Hg} 3-\mathrm{I} 5=2.617(1) \\
\mathrm{Hg} 1-\mathrm{I} 6=2.635(1) \\
\mathrm{Hg} 1-\mathrm{I} 2=2.648(1) \\
\mathrm{Hg} 1-\mathrm{I} 1=3.080(1) \\
\mathrm{Hg} 1-\mathrm{I} 8=3.207(1) \\
\mathrm{Hg} 2-\mathrm{I} 3=2.715(1) \\
\mathrm{Hg} 2-\mathrm{I} 9=2.749(1) \\
\mathrm{Hg} 2-\mathrm{I} 10=2.855(1) \\
\mathrm{Hg} 2-\mathrm{I} 7=2.910(1) \\
\mathrm{Hg} 3-\mathrm{I} 4=2.623(1) \\
\mathrm{Hg} 3-\mathrm{I} 9=3.153(1) \\
\mathrm{Hg} 3-\mathrm{I} 3=3.315(1) \\
\mathrm{Hg} 4-\mathrm{I} 8=2.744(1) \\
\mathrm{Hg} 4-\mathrm{I} 1=2.766(1) \\
\mathrm{Hg} 4-\mathrm{I} 7=2.835(1) \\
\mathrm{Hg} 4-\mathrm{I} 10=2.876(1)\end{array}$ & $86.82-149.08$ \\
\hline
\end{tabular}




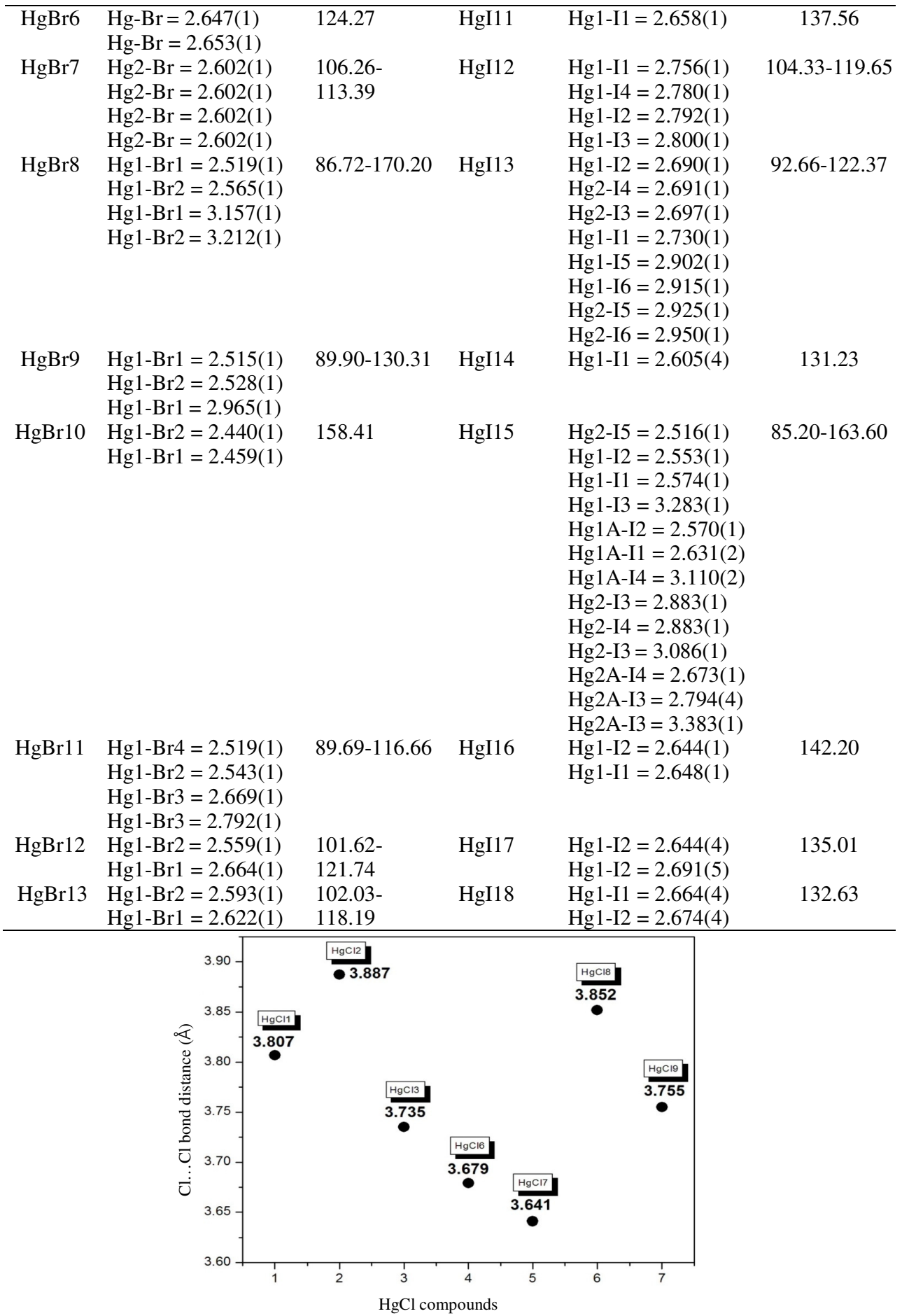

Figure 2. Scatter plot for $\mathrm{Cl}-\mathrm{Cl}$ bond distances 
Table 3. Halogen...Halogen bond distances $(\AA)$ and torsion angle $\left(^{\circ}\right)$ in $[\mathrm{HgX}]^{-},(\mathrm{X}=\mathrm{Cl}, \mathrm{Br}, \mathrm{I})$ compounds with symmetry positions

\begin{tabular}{|c|c|c|c|c|c|}
\hline Code & $\begin{array}{l}\text { X...X bond } \\
\text { distance }(\AA)\end{array}$ & $\begin{array}{c}\text { Torsion Angle } \\
{[1,2,3,4]^{\circ}}\end{array}$ & Code & $\begin{array}{l}\text { X...X bond } \\
\text { distance }(\AA)\end{array}$ & $\begin{array}{c}\text { Torsion Angle } \\
{[1,2,3,4]^{\circ}}\end{array}$ \\
\hline $\mathrm{HgCl1}$ & $\begin{array}{c}\mathrm{Cl} 2 \ldots \mathrm{Cl} 2= \\
3.807(4)\end{array}$ & 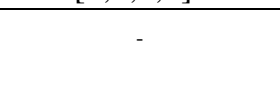 & $\mathrm{HgBr} 14$ & $\begin{array}{c}\mathrm{Br} 1 \ldots \mathrm{Br} 1= \\
4.921(1)\end{array}$ & $\begin{array}{l}\mathrm{Hg} 1-\mathrm{Br} 1 \ldots \mathrm{Br} 1^{\text {xix }}- \\
\mathrm{Hg} 1^{\text {xix }}=180.00(2)\end{array}$ \\
\hline $\mathrm{HgCl} 2$ & $\begin{array}{l}\mathrm{Cl} \ldots \mathrm{Cl}= \\
3.887(1)\end{array}$ & - & $\mathrm{HgBr} 15$ & $\begin{array}{c}\mathrm{Br} 1 \ldots \mathrm{Br} 2= \\
4.479(1)\end{array}$ & $\begin{array}{l}\mathrm{Hg} 1-\mathrm{Br} 2 \ldots \mathrm{Br} 1^{\mathrm{xx}} \\
\mathrm{Hg} 1^{\mathrm{xx}}=34.57(3)\end{array}$ \\
\hline $\mathrm{HgCl} 3$ & $\begin{array}{c}\mathrm{Cl}(1) \ldots \mathrm{Cl}(2) \\
=3.735(1) \\
\mathrm{Cl}(2) \ldots \mathrm{Cl}(2) \\
=3.919(1)\end{array}$ & $\begin{array}{c}\mathrm{Hg}-\mathrm{Cl}(1) \ldots \mathrm{Cl}^{\mathrm{i}}(2)- \\
\mathrm{Hg}^{\mathrm{i}}=79.95(2)\end{array}$ & $\mathrm{HgBr} 16$ & $\begin{array}{c}\mathrm{Br} 1 \ldots \mathrm{Br} 3= \\
3.822(1)\end{array}$ & $\begin{array}{l}\mathrm{Hg} 1-\mathrm{Br} 2 \ldots \mathrm{Br} 4^{\mathrm{xxi}} \\
\mathrm{Hg} 2^{\mathrm{xxi}}=110.22(2)\end{array}$ \\
\hline $\mathrm{HgCl} 4$ & $\begin{array}{c}\mathrm{Cl} 1 \ldots \mathrm{Cl} 2= \\
4.245(4)\end{array}$ & $\begin{array}{c}\mathrm{Hg}-\mathrm{Cl} 4 \ldots \mathrm{Cl} 3-\mathrm{Hg}^{\mathrm{ii}} \\
=-89.41(2)\end{array}$ & $\operatorname{HgBr} 17$ & $\begin{array}{c}\operatorname{Br} 1 \ldots \mathrm{Br} 1= \\
4.186(1)\end{array}$ & $\begin{array}{c}\mathrm{Hg} 1-\mathrm{Br} 1 \ldots \mathrm{Br} 1^{\mathrm{xii}}- \\
\mathrm{Hg}^{\mathrm{xxii}}=-180.00(2)\end{array}$ \\
\hline $\mathrm{HgCl} 5$ & $\begin{array}{c}\mathrm{Cl} 1 \ldots \mathrm{Cl} 2= \\
4.470(5)\end{array}$ & $\begin{array}{c}\mathrm{Hg} g-\mathrm{Cl} 1 \ldots \mathrm{Cl} 3^{\mathrm{iii}}- \\
\mathrm{Hg}^{\mathrm{iii}}=-123.04(2)\end{array}$ & $\mathrm{HgBr} 18$ & $\begin{array}{c}\mathrm{Br} 1-\mathrm{Br} 1= \\
5.296(3)\end{array}$ & $\begin{array}{c}\mathrm{Hg} 1-\mathrm{Br} 1 \ldots \mathrm{Br} 1^{\text {xxiii }}- \\
\mathrm{Hg} 1^{\text {xxiii }}=0.00(5)\end{array}$ \\
\hline $\mathrm{HgCl} 6$ & $\begin{array}{c}\mathrm{Cl} 2 \ldots \mathrm{Cl} 3= \\
3.679(2) \\
\mathrm{Cl1} \ldots \mathrm{Cl} 3= \\
3.797(2) \\
\mathrm{Cl} 2 \ldots \mathrm{Cl} 1= \\
3.853(2)\end{array}$ & 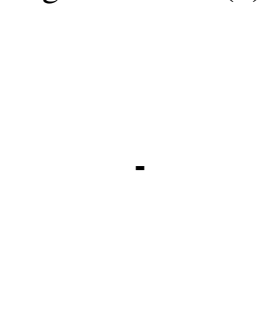 & HgI1 & $\begin{array}{l}\mathrm{I} 7 \ldots \mathrm{I} 7= \\
2.737(4) \\
\mathrm{I} 4 \ldots \mathrm{I} 5= \\
2.821(3) \\
\mathrm{I} 5 \ldots \mathrm{I} 6= \\
2.979(3) \\
\mathrm{I} 6 \ldots \mathrm{I} 7= \\
3.421(3)\end{array}$ & 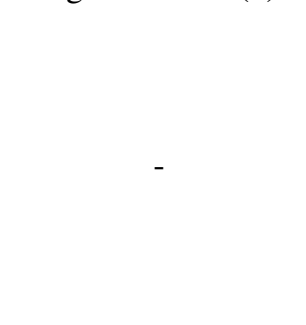 \\
\hline $\mathrm{HgCl} 7$ & $\begin{array}{c}\mathrm{Cl} 4 \ldots \mathrm{Cl} 4= \\
3.641(3) \\
\mathrm{Cl} 2 \ldots \mathrm{Cl} 3= \\
3.839(3)\end{array}$ & - & $\mathrm{HgI} 2$ & $\begin{array}{l}\mathrm{I} 1 \ldots \mathrm{I} 1= \\
4.149(2)\end{array}$ & $\begin{array}{c}\mathrm{Hg} 1-\mathrm{I} 1 \ldots \mathrm{I} 1^{\mathrm{xxiv}}- \\
\mathrm{Hg} 1^{\mathrm{xxv}}=-180.00(1)\end{array}$ \\
\hline $\mathrm{HgCl} 8$ & $\begin{array}{c}\mathrm{Cl} 1 \ldots \mathrm{Cl} 3= \\
3.852(1)\end{array}$ & $\begin{array}{c}\mathrm{Hg}_{-} \mathrm{Cl} 3 \ldots \mathrm{Cl} 1^{\mathrm{iv}}- \\
\mathrm{Hg}^{\mathrm{iv}}=175.79(4)\end{array}$ & $\mathrm{HgI} 3$ & $\begin{array}{l}\mathrm{I} 2 \ldots \mathrm{I} 1= \\
4.360(6)\end{array}$ & - \\
\hline $\mathrm{HgCl} 9$ & 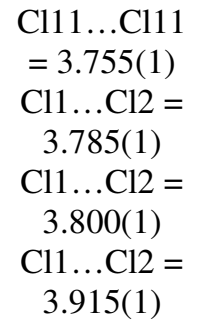 & $\begin{array}{l}\mathrm{Hg} 1-\mathrm{Cl} 11 \ldots \mathrm{Cl} 11^{\mathrm{v}}- \\
\mathrm{Hg} 1^{\mathrm{v}}=-180.00(3)\end{array}$ & $\mathrm{HgI} 4$ & $\begin{array}{l}\mathrm{I} 3 \ldots \mathrm{I} 3= \\
4.215(2)\end{array}$ & $\begin{array}{c}\mathrm{Hg}-\mathrm{I} 3 \ldots \mathrm{I} 3^{\mathrm{xxvi}}-\mathrm{Hg}^{\mathrm{xxvi}} \\
=68.17(2)\end{array}$ \\
\hline $\mathrm{HgCl10}$ & $\begin{array}{c}\mathrm{Cl} 3 \ldots \mathrm{Cl} 4= \\
5.601(2)\end{array}$ & $\begin{array}{c}\mathrm{Hg} 1-\mathrm{Cl} 4 \ldots \mathrm{Cl} 3^{\mathrm{vi}}- \\
\mathrm{Hg} 1^{\mathrm{vi}}=-103.35(5)\end{array}$ & HgI5 & $\begin{array}{l}\mathrm{I} 1 \ldots \mathrm{I} 2= \\
7.337(3)\end{array}$ & $\begin{array}{c}\mathrm{Hg}-\mathrm{I} 2 \ldots \mathrm{I}^{\mathrm{xxvii}}-\mathrm{Hg}^{\mathrm{xxvi}} \\
=-43.36(1)\end{array}$ \\
\hline $\mathrm{HgBr} 1$ & $\begin{array}{c}\mathrm{Br} 2 \ldots \mathrm{Br} 3= \\
3.610(2)\end{array}$ & $\begin{array}{l}\mathrm{Hg}-\mathrm{Br} 2 \ldots \mathrm{Br} 3^{\mathrm{vl1}}- \\
\mathrm{Hg}^{\text {vii }}=121.58(1)\end{array}$ & HgI6 & $\begin{array}{l}\mathrm{I} 2 \ldots \mathrm{I} 2= \\
4.492(1)\end{array}$ & $\begin{array}{c}\mathrm{Hg}-\mathrm{I} 2 \ldots 2^{\text {xvv11 }}- \\
\mathrm{Hg}^{\text {xxviii }}=179.18(1)\end{array}$ \\
\hline $\mathrm{HgBr} 2$ & $\begin{array}{c}\mathrm{Br} \ldots \mathrm{Br}= \\
4.356(1)\end{array}$ & $\begin{array}{c}\mathrm{Hg}-\mathrm{Br} . . . \mathrm{Br}^{\mathrm{vHII}}- \\
\mathrm{Hg}^{\mathrm{viii}}=-51.14(2)\end{array}$ & $\mathrm{HgI7}$ & $\begin{array}{l}\mathrm{I} 2 \ldots \mathrm{I} 2= \\
4.134(2)\end{array}$ & $\begin{array}{c}\mathrm{Hg}-\mathrm{I} 2 \ldots \mathrm{I}^{\mathrm{xxIx}}-\mathrm{Hg}^{\mathrm{xxix}} \\
=67.55(1)\end{array}$ \\
\hline $\mathrm{HgBr} 3$ & $\begin{array}{c}\operatorname{Br} 1 \ldots \mathrm{Br} 1= \\
4.138(2)\end{array}$ & $\begin{array}{c}\mathrm{Hg}-\mathrm{Br} 1 \ldots \mathrm{Br} 1^{\mathrm{ix}}- \\
\mathrm{Hg}^{\mathrm{Ix}}=-180.00(3)\end{array}$ & HgI8 & $\begin{array}{l}\mathrm{I} 1 \ldots \mathrm{I} 2= \\
4.121(2)\end{array}$ & $\begin{array}{c}\mathrm{Hg}-\mathrm{I} 2 \ldots \mathrm{I}^{\mathrm{xxx}}-\mathrm{Hg}^{\mathrm{xxx}} \\
=67.48(4)\end{array}$ \\
\hline $\mathrm{HgBr} 4$ & $\begin{array}{c}\mathrm{Br} 3 \ldots \mathrm{Br} 3= \\
3.915(2)\end{array}$ & $\begin{array}{l}\mathrm{Hg} 1-\mathrm{Br} 3 \ldots \mathrm{Br} 3^{\mathrm{x}}- \\
\mathrm{Hg} 1=180.00(5)\end{array}$ & HgI9 & $\begin{array}{l}\mathrm{I} 2 \ldots \mathrm{I} 3= \\
4.137(2)\end{array}$ & $\begin{array}{c}\mathrm{Hg}-\mathrm{I} 2 \ldots 3^{\mathrm{xxx11}}- \\
\mathrm{Hg}^{\mathrm{xxxiii}}=67.96(1)\end{array}$ \\
\hline
\end{tabular}




\begin{tabular}{|c|c|c|c|c|c|}
\hline $\mathrm{HgBr} 5$ & $\begin{array}{c}\mathrm{Br} 1 \ldots \mathrm{Br} 4= \\
3.662(2) \\
\mathrm{Br} 1 \ldots \mathrm{Br} 1= \\
3.992(2)\end{array}$ & $\begin{array}{c}\mathrm{Hg}-\mathrm{Br} 4 \ldots \mathrm{Br} 1^{\mathrm{xi}}- \\
\mathrm{Hg} 1^{\mathrm{xi}}=145.00(1)\end{array}$ & HgI10 & $\begin{array}{l}\mathrm{I} 3 \ldots \mathrm{I} 3= \\
4.343(1)\end{array}$ & $\begin{array}{c}\mathrm{Hg} 2-\mathrm{I} 3 \ldots \mathrm{I} 3^{\text {xxxiv }}- \\
\mathrm{Hg} 2^{\text {xxxiv }}=-180.00(5)\end{array}$ \\
\hline $\mathrm{HgBr6}$ & $\begin{array}{l}\mathrm{Br} \ldots \mathrm{Br}= \\
4.662(1)\end{array}$ & $\begin{array}{c}\mathrm{Hg}-\mathrm{Br} \ldots \mathrm{Br}^{\mathrm{xii}}- \\
\mathrm{Hg}^{\mathrm{xiii}}=2.45(5)\end{array}$ & HgI11 & $\begin{array}{l}\mathrm{I} 1 \ldots \mathrm{I} 1= \\
5.667(2)\end{array}$ & $\begin{array}{c}\mathrm{Hg} 1-\mathrm{I} 1 \ldots \mathrm{I} 1^{\mathrm{xxxv}}- \\
\mathrm{Hg} 1^{\mathrm{xxxv}}=180.00(3)\end{array}$ \\
\hline $\mathrm{HgBr} 7$ & $\begin{array}{l}\mathrm{Br} \ldots \mathrm{Br}= \\
5.817(2)\end{array}$ & $\begin{array}{c}\mathrm{Hg} 2-\mathrm{Br} \ldots \mathrm{Br}^{\mathrm{xiv}}- \\
\mathrm{Hg} 2^{\text {xiv }}=-61.76(7)\end{array}$ & HgI12 & $\begin{array}{l}\mathrm{I} 2 \ldots \mathrm{I} 4= \\
6.459(1)\end{array}$ & $\begin{array}{c}\operatorname{Hg} 1-\mathrm{I} 4 \ldots . . \mathrm{I} 2^{\text {xxxvi }}- \\
\mathrm{Hg} 1^{\text {xxxvi }}=-164.47(2)\end{array}$ \\
\hline $\mathrm{HgBr} 8$ & $\begin{array}{c}\operatorname{Br} 1 \ldots \mathrm{Br} 1= \\
3.925(1)\end{array}$ & $\begin{array}{l}\mathrm{Hg} 1-\mathrm{Br} 1 \ldots \mathrm{Br}^{\mathrm{xv}}- \\
\mathrm{Hg} 1^{\mathrm{xv}}=170.71(2)\end{array}$ & HgI13 & $\begin{array}{l}\mathrm{I} 5 \ldots \mathrm{I} 6= \\
4.249(1)\end{array}$ & $\begin{array}{l}\mathrm{Hg} 2-\mathrm{I} 5 \ldots \mathrm{I} 6-\mathrm{Hg} 1^{\mathrm{xxxvii}} \\
=-175.63(3)\end{array}$ \\
\hline $\mathrm{HgBr} 9$ & $\begin{array}{c}\operatorname{Br} 1 \ldots \mathrm{Br} 1= \\
3.891(2) \\
\operatorname{Br} 1 \ldots \mathrm{Br} 2= \\
3.982(2)\end{array}$ & $\begin{array}{c}\mathrm{H} 1-\mathrm{Br} 1 \ldots \mathrm{Br} 1^{\mathrm{xvi}}- \\
\mathrm{Hg} 1^{\mathrm{xvi}}=180.00(5)\end{array}$ & HgI14 & $\begin{array}{l}\mathrm{I} 1 \ldots \mathrm{I} 1= \\
7.332(1)\end{array}$ & $\begin{array}{c}\operatorname{Hg} 1-\mathrm{I} 1 \ldots \mathrm{I} 1^{\text {xxxviii }} \\
\mathrm{Hg} 1^{\text {xxxviii }}=180.00(1)\end{array}$ \\
\hline $\mathrm{HgBr} 10$ & $\begin{array}{c}\mathrm{Br} 2 \ldots \mathrm{Br} 2= \\
3.956(1)\end{array}$ & $\begin{array}{c}\mathrm{Hg} 1-\mathrm{Br} 2 \ldots \mathrm{Br} 2^{\mathrm{xvii}}- \\
\operatorname{Hg} 1^{\mathrm{xvii}}=- \\
124.06(3)\end{array}$ & HgI15 & $\begin{array}{c}\mathrm{I} 1-\mathrm{I} 1= \\
3.811(2)\end{array}$ & $\begin{array}{c}\operatorname{Hg} 1-I 1 \ldots I^{\text {xxxix }} \\
\operatorname{Hg} 1 A^{\text {xxxix }}=- \\
156.65(1)\end{array}$ \\
\hline $\mathrm{HgBr} 11$ & $\begin{array}{c}\mathrm{Br} 3 \ldots \mathrm{Br} 3= \\
3.852(1)\end{array}$ & $\begin{array}{c}\mathrm{Hg} 1-\mathrm{Br} 3 \ldots \mathrm{Br} 3^{\text {xviii }} \\
\mathrm{Hg} 1=-180.00(2)\end{array}$ & HgI16 & $\begin{array}{l}\mathrm{I} 1 \ldots \mathrm{I} 2= \\
4.594(1)\end{array}$ & $\begin{array}{l}\mathrm{Hg} 1-\mathrm{I} 1 \ldots \mathrm{I} 2^{\mathrm{xl}}-\mathrm{Hg}^{\mathrm{xl}} \\
=-37.52(2)\end{array}$ \\
\hline $\mathrm{HgBr} 12$ & $\begin{array}{c}\mathrm{Br} 2 \ldots \mathrm{Br} 2= \\
4.083(2)\end{array}$ & - & HgI17 & $\begin{array}{l}\mathrm{I} 1 \ldots \mathrm{I} 2= \\
4.476(6)\end{array}$ & $\begin{array}{l}\operatorname{Hg} 1-\mathrm{I} 2 \ldots \mathrm{II}^{\mathrm{xli}}-\mathrm{Hg} 1^{\mathrm{xli}} \\
\quad=-134.14(2)\end{array}$ \\
\hline $\mathrm{HgBr} 13$ & $\begin{array}{c}\mathrm{Br} 1 \ldots \mathrm{Br} 3= \\
3.396(1)\end{array}$ & - & HgI18 & $\begin{array}{l}\mathrm{I} 1 \ldots \mathrm{I} 2= \\
4.197(5)\end{array}$ & $\begin{array}{c}\mathrm{Hg} 1-\mathrm{I} 1 \ldots \mathrm{I} 2^{\mathrm{xlii}}-\mathrm{Hg} 1^{\mathrm{xlii}} \\
=-176.07(2)\end{array}$ \\
\hline
\end{tabular}

Symmetry codes: (i) $\mathrm{x}, 1-\mathrm{y},-0.5+\mathrm{z}$ (ii) $0.5-\mathrm{x}, 0.5+\mathrm{y}, 0.5-\mathrm{z}$ (iii) $-\mathrm{x}, 0.5+\mathrm{y}, 1-\mathrm{z}$ (iv) $\mathrm{x},-1+\mathrm{y}, \mathrm{z}$ (v) -x, $\mathrm{y},-\mathrm{z}$ (vi) $-1+\mathrm{x}, \mathrm{y}, \mathrm{z}$ (vii) $\mathrm{x}, 0.5-\mathrm{y}, 0.5+\mathrm{z}$ (viii) $0.5+\mathrm{x}, 1-\mathrm{y}, \mathrm{z}$ (ix) -x, -y, 1-z (x) -x, 1-y, -z (xi) x, -y, $0.5-(x i i) 1-x, y, 0.5-z$ (xiii) $0.5+x,-0.5+y, z$ (xiv) $-0.25-x,-0.25-y, z$ (xv) 1-x, y, 0.5-z (xvi) 1-x, 2-y, 1-z (xvii) 1-x, y, 0.5-z (xviii) 1-x, -y, 2-z (xix) -1-x, 1-y, -z (xx) 1-x, 1-y, 3-z (xxi) 1-x, -y, 1-z (xxii) 1-x, 1-y, -z (xxiii) x, 1+y, z (xxiv) -x, -y, 1-z (xxv) x, -0.5+y, 1-z (xxvi) 2-x, y, 0.5-z (xxvii) 0.5+x, $1-y, z$ (xxviii) -x, 0.5+y, -z (xxix) $-0.25+y, 0.25+x, 0.25-z$ (xxx) $1.5-x, y, 0.5-z$ (xxxi) $1.25-y,-$ $0.25+x, 0.25+z$ (xxxii) 1-x, -0.5+y, 1-z (xxxiii) $0.5-x, y, 0.5+z$ (xxxiv) 1-x, 1-y, 1-z (xxxv) -x, 1-y, z xxxvi) 1.5-x, 0.5+y, 1.5-z (xxxvii) x, 0.5-y. 0.5+z (xxxviii) x, -1+y, z (xxxix) 0.5-x, -0.5-y, 1-z (xl) 1-x, 1-y, 3-z (xli) x, 1+y, z (xlii) $-0.5+x,-0.5+y, z$

\section{[HgBr] Hybrid materials}

The bond lengths of $\mathrm{Hg}-\mathrm{Br}$ bond lies in an average range of $2.401 \AA$ to $2.669 \AA$. The graphical projection of bond distances for these derivatives illustrate that most of the data points lie in between 2.45 to $2.70 \AA$ whereas the data points for $\mathrm{HgBr} 2, \mathrm{HgBr} 3, \mathrm{HgBr} 8$ and $\mathrm{HgBr} 14$ shows the deviation from this linearity as presented in Figure 3. The $\mathrm{Br}-\mathrm{Hg}-\mathrm{Br}$ bond angles have wide range from minimum value of 126.49 to $132.85^{\circ}$ for molecule $\mathrm{HgBr} 16$ and maximum range of 92.84 to $180^{\circ}$ for molecule $\mathrm{HgBr} 5$ as described in Table 2 .

The scatter plot for $\mathrm{Br} . . . \mathrm{Br}$ bond distances as shown in Figure 4 illustrates that most of the data points exist in the range of $3.610 \AA$ to $3.922 \AA$ which shows that the crystal structures are stabilized by secondary interactions as this range is comparable with van der Waals radii. Among all these compounds showing Halogen...Halogen interactions, $\mathrm{HgBr} 8$ has the lowest value of $3.396 \AA$. 


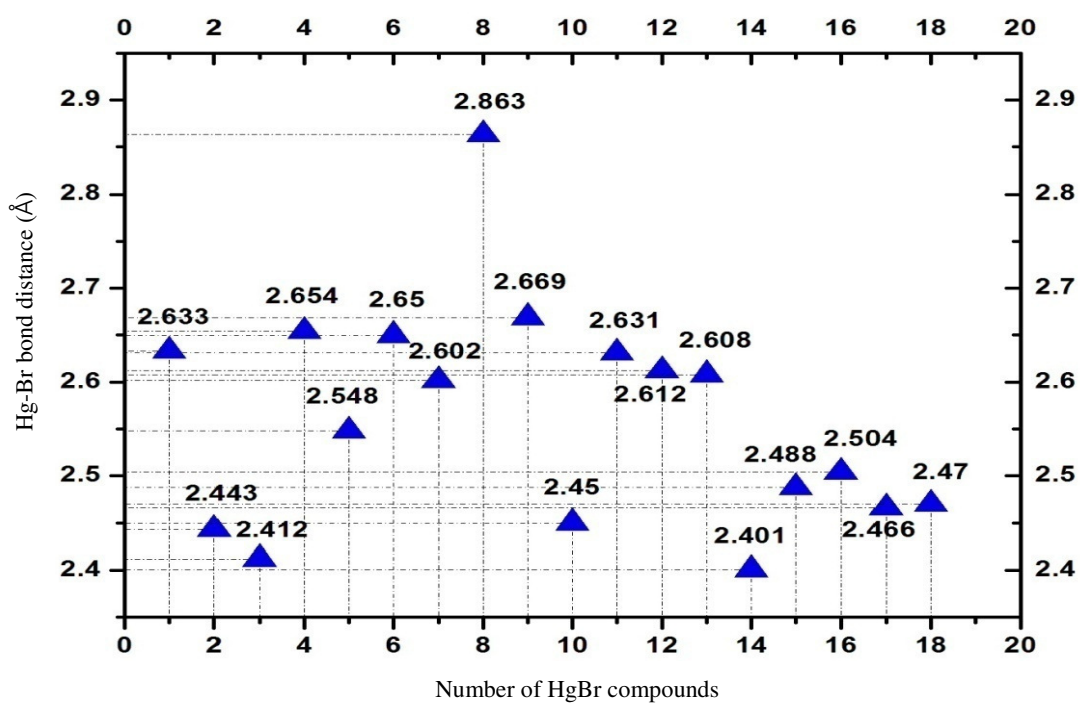

Figure 3. Scatter Plot of the average values of $\mathrm{Hg}-\mathrm{Br}$ compounds

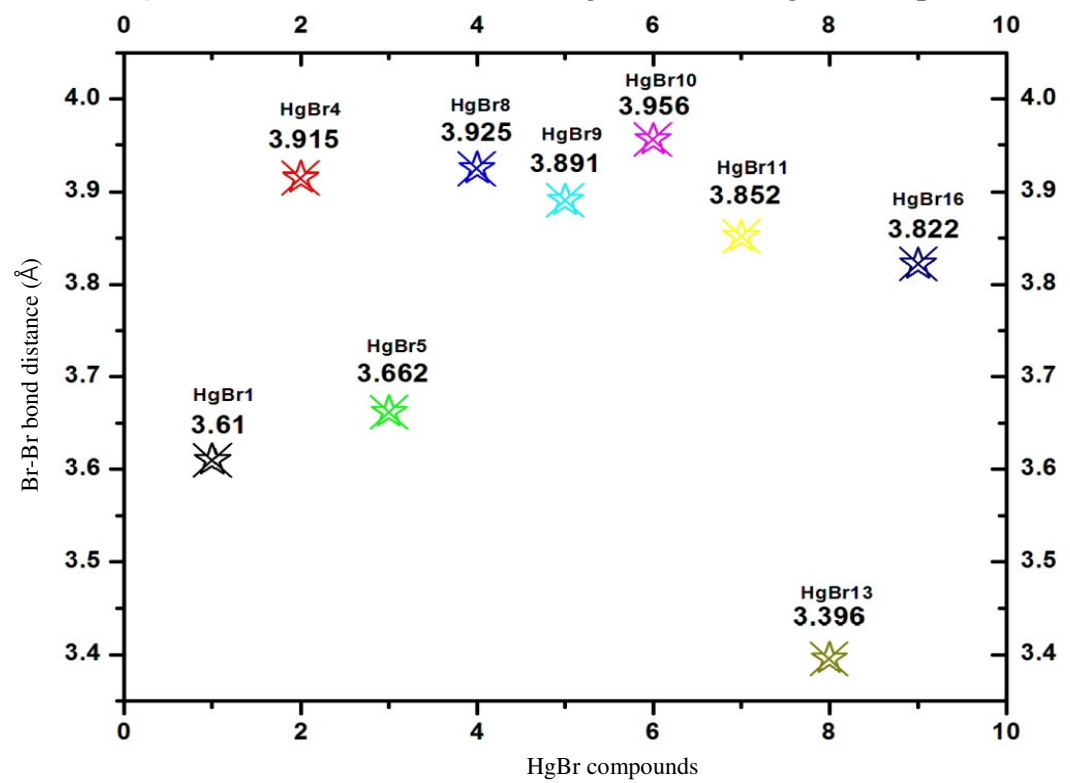

Figure 4. Scatter plot for $\mathrm{Br}-\mathrm{Br}$ bond distances

\section{[HgI] Hybrid materials}

The bond lengths of Hg-I bond lies in an average range of $2.605 \AA$ to $2.785 \AA$. The graphical projection of bond distances for these derivatives illustrate that most of the data points lie in between 2.65 to $2.80 \AA$ whereas the data points for $\mathrm{HgI} 8, \mathrm{HgI} 12, \mathrm{HgI} 13, \mathrm{HgI} 14$ and $\mathrm{HgI} 15$ shows the deviation from this linearity as presented in Figure 5. The I-Hg-I bond angles have wide range from minimum value of 107.74 to $112.98^{\circ}$ for molecule $\mathrm{HgI} 2$ and maximum range of 85.20 to $163.60^{\circ}$ for molecule $\mathrm{HgI} 15$ as shown in Table 2 . 


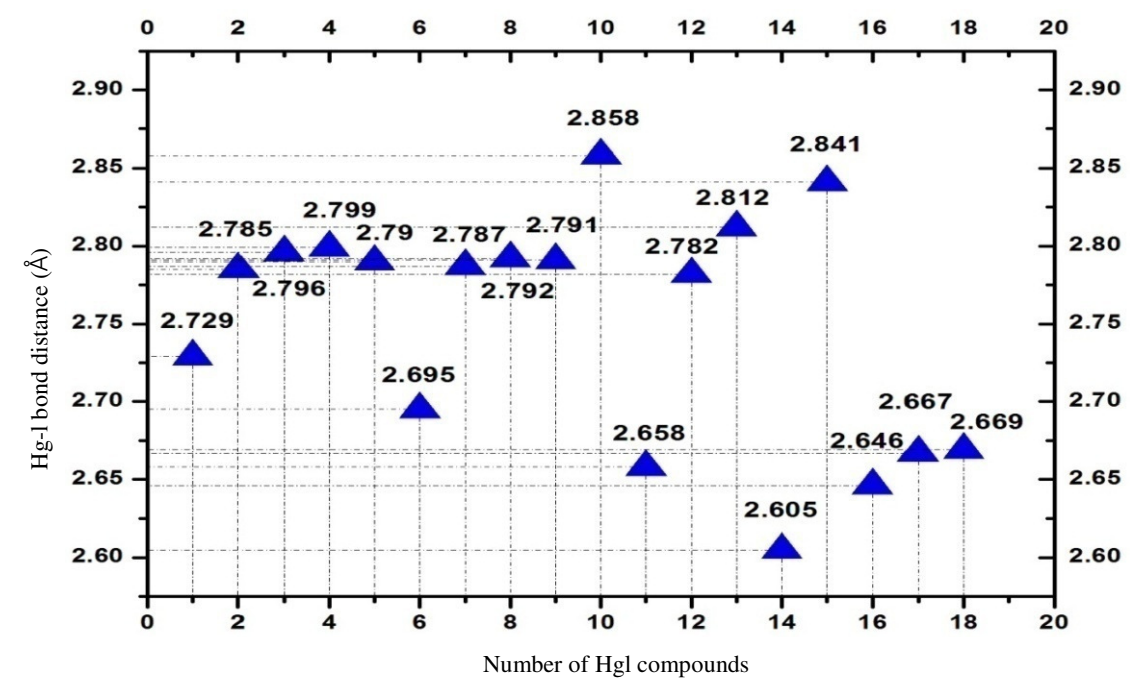

Figure 5. Scatter Plot of the average values of $\mathrm{Hg}-\mathrm{I}$ bond distances.

While calculating the Halogen...Halogen Interactions based on $\mathrm{HgI}$ derivatives, it is found that only two derivatives i.e. HgI1 and HgI13 shows the minimum values of $3.420 \AA$ and $3.835 \AA$ respectively. All other compounds show its minimum values in the range of $4.121 \AA$ to $4.956 \AA$ which are not comparable with Van der Waals radii and hence cannot be fitted for Halogen...Halogen interactions (Table 3). The minimum value of torsion angle [I$\mathrm{Hg} \ldots \mathrm{Hg}-\mathrm{I}]$ is $2.15(3)^{\circ}$ for the compound $\mathrm{HgIl} 1$ at $1-\mathrm{x}, 1-\mathrm{y}, 1-\mathrm{z}$ and $\mathrm{x}, 0.5-\mathrm{y}, 0.5+\mathrm{z}$ symmetry positions, while its maximum value is 180.00 (1) for $\mathrm{HgI} 12$ with $\mathrm{x},-1+\mathrm{y}, \mathrm{z}$ symmetry positions as given in Table 3 .

The $\mathrm{Hg}$...Hg distance in compound $\mathrm{HgCl} 1$ is calculated as 3.831(1) $\AA$ which shows that the structure is stabilized by metallophilic interactions apart from $\mathrm{X}-\mathrm{H}$... A and $\mathrm{Cl}$... Cl secondary interactions. Similar pattern of mercurophilic interactions is observed in $\mathrm{HgCl} 2$ derivative with $\mathrm{Hg}$... Hg distance of $3.920(2) \AA$. $\mathrm{In} \mathrm{HgCl} 3$ derivative, $\mathrm{Hg}$ atom at symmetry position $\mathrm{x}, 1+\mathrm{y},-1+\mathrm{z}$ establish a close contact of 3.810(3) $\AA$ with another $\mathrm{Hg}$ atom at symmetry position $1-\mathrm{x}, 1+\mathrm{y}, 0.5$ z. The pictorial projection is plotted along ac-plane and $1 \mathrm{D}$ chain pattern of $\mathrm{Hg}$...Hg contacts

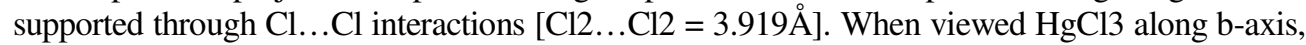
the organic layers are held in between this zig-zag pattern of inorganic halides through $\mathrm{N}-\mathrm{H}$...Cl interactions, in which $\mathrm{Cl} 1$ and $\mathrm{Cl} 2$ are responsible for stabilizing the organic-inorganic moieties at symmetry positions $\mathrm{x},-\mathrm{y},-0.5+\mathrm{z}$ and $1-\mathrm{x},-\mathrm{y}, 1-\mathrm{z}$ respectively. $\mathrm{Cl} 2$ at symmetry position $1-\mathrm{x},-$ $\mathrm{y}, 1-\mathrm{z}$ acts as bifurcated hydrogen acceptors having bifurcated angle as $92.24(2)^{\circ}$. The organic moiety (Phenylpiperazinium) stacked within the inorganic layers is further stabilized by $\mathrm{C}-\mathrm{H} \ldots \pi$ interactions at $1.5-\mathrm{x},-0.5+\mathrm{y}, 0.5-\mathrm{z}$ where $\mathrm{H}-\pi=2.983(1)$ and $\mathrm{C}-\mathrm{H} \ldots \pi=133.8^{\circ}$. The inorganic part (i.e. trichloromercurate) of the hybrid materials stabilized through zig-zag pattern of secondary interactions and the organic part (i.e. phenylpiperazinium) is sandwiched between inorganic layers as shown in Figure 6 along $a c$-plane.

The $\mathrm{Hg}$...Hg distance in compound $\mathrm{HgBr} 1$ is calculated as 3.970(1) $\mathrm{A}$ which shows that the structure is stabilized by metallophilic interactions apart from X-H...A and $\mathrm{Br}$...Br [minimum distance $=3.610(2) \AA$ ] secondary interactions ${ }^{10,19}$. Mercury atom at symmetry position $1+\mathrm{x}, 0.5-\mathrm{y}$, and $0.5+\mathrm{z}$ establish a close contact with $\mathrm{Hg}$ located at $1-\mathrm{x}, 0.5+\mathrm{y}$, and $0.5-\mathrm{z}$ as shown in Figure 7. 


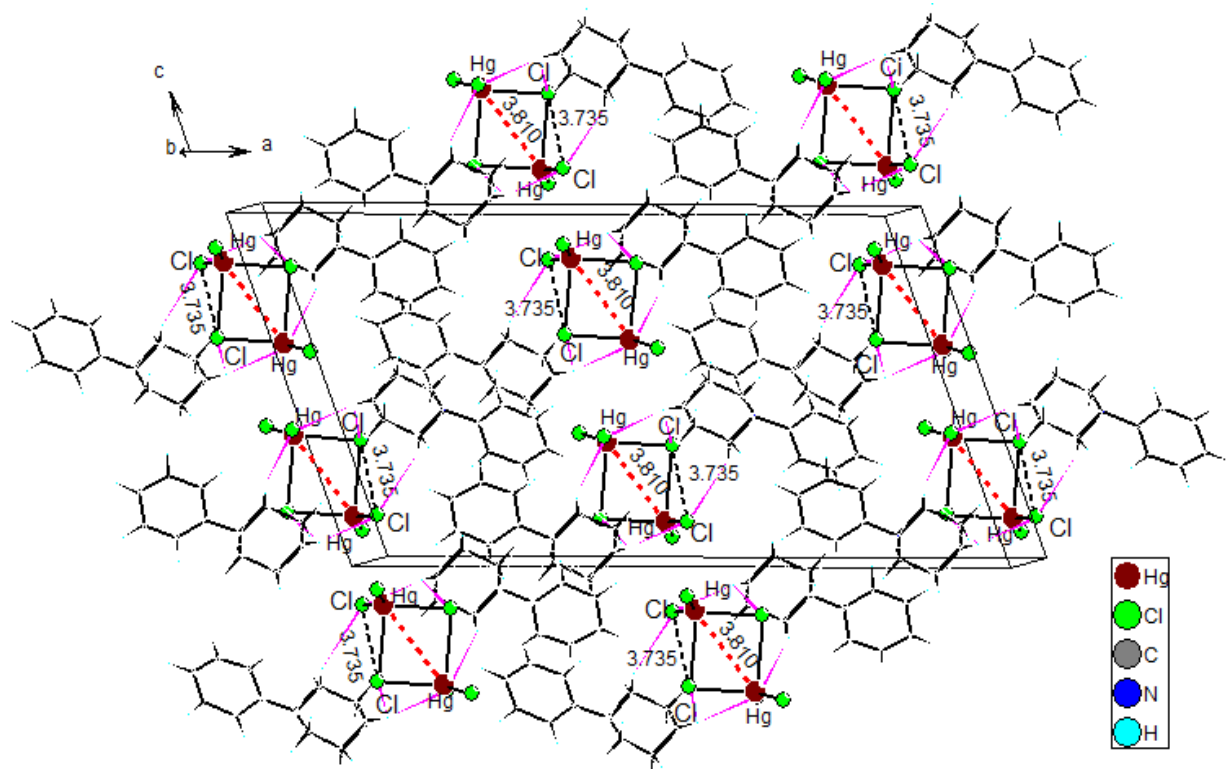

Figure 6. $1 \mathrm{D}$ chain pattern of $\mathrm{Hg} \ldots \mathrm{Hg}$ and $\mathrm{Cl} \ldots \mathrm{Cl}$ interactions in Phenylpiperazinium trichloromercurate

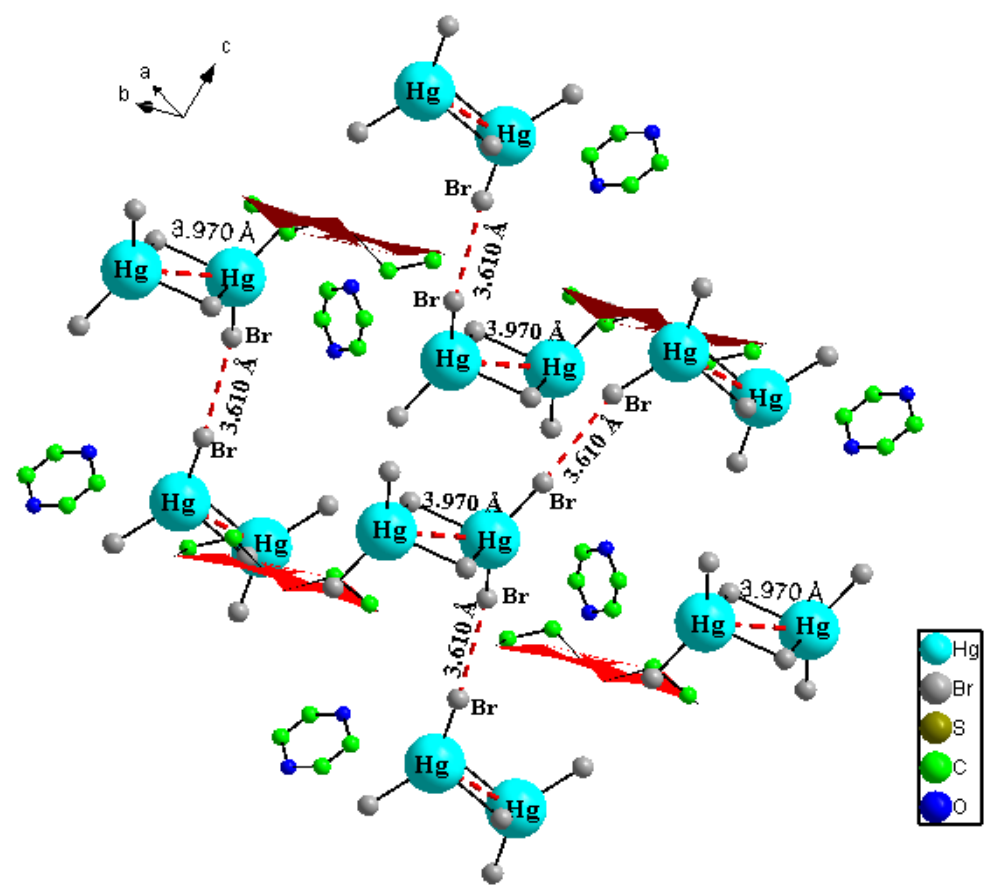

Figure 7. $\mathrm{Hg} . . . \mathrm{Hg}$ and $\mathrm{Br} . . \mathrm{Br}$ interactions in $\mathrm{HgBr} 1$ derivatives

IR and Raman tensors

IR and Raman spectra tensors have been calculated by using fractional co-ordinate obtained from single crystal XRD data as presented in Table 4. 
Table 4. Infra-Red and Raman spectra tensors for $\mathrm{HgX}$ derivatives

\begin{tabular}{|c|c|c|c|c|c|}
\hline Compound & Active Mode Equation & Wykoff Positions & $\begin{array}{l}\text { Infra- } \\
\text { Red }\end{array}$ & Raman & $\begin{array}{l}\text { Hyper- } \\
\text { Raman }\end{array}$ \\
\hline $\mathrm{HgCl} 12$ & $\left(\Gamma_{\text {acoustic }}=3 \mathrm{~A}_{\mathrm{u}}\right)$ & WP1 =[2i $]$ & $\mathrm{A}_{\mathrm{u}}=1$ & $\mathrm{~A}_{\mathrm{g}}=1$ & $\mathrm{~A}_{\mathrm{u}}=1$ \\
\hline $\mathrm{HgCl} 3$ & $\left(\Gamma_{\text {acoustic }}=A_{u}+2 B_{u}\right)$ & $\mathrm{WP} 1=[8 \mathrm{f}]$ & $\begin{array}{l}\mathrm{A}_{\mathrm{u}}=1 \\
\mathrm{~B}_{\mathrm{u}}=1\end{array}$ & $\begin{array}{l}A_{g}=1 \\
B_{g}=1\end{array}$ & $\begin{array}{l}\mathrm{A}_{\mathrm{u}}=1 \\
\mathrm{~B}_{\mathrm{u}}=1\end{array}$ \\
\hline $\mathrm{HgCl} 4$ & $\left(\Gamma_{\text {acoustic }}=A_{u}+2 B_{u}\right)$ & $\mathrm{WP} 1=[4 \mathrm{e}]$ & $\begin{array}{l}\mathrm{A}_{\mathrm{u}}=1 \\
\mathrm{~B}_{\mathrm{u}}=1\end{array}$ & $\begin{array}{l}A_{g}=1 \\
B_{g}=1\end{array}$ & $\begin{array}{l}\mathrm{A}_{\mathrm{u}}=1 \\
\mathrm{~B}_{\mathrm{u}}=1\end{array}$ \\
\hline $\mathrm{HgCl} 5$ & $\left(\Gamma_{\text {acoustic }}=\mathrm{A}+2 \mathrm{~B}\right)$ & $\mathrm{WP} 1=[2 \mathrm{a}]$ & $\begin{array}{l}A=1 \\
B=1\end{array}$ & $\begin{array}{l}A=1 \\
B=1\end{array}$ & $\begin{array}{l}A=1 \\
B=1\end{array}$ \\
\hline $\mathrm{HgCl} 6$ & $\left(\Gamma_{\text {acoustic }}=3 \mathrm{~A}_{\mathrm{u}}\right)$ & $\mathrm{WP} 3=[1 \mathrm{a}, 1 \mathrm{~h}, 2 \mathrm{i}]$ & $\mathrm{A}_{\mathrm{u}}=3$ & $\mathrm{~A}_{\mathrm{g}}=1$ & $\mathrm{~A}_{\mathrm{u}}=3$ \\
\hline $\mathrm{HgCl} 7$ & $\left(\Gamma_{\text {acoustic }}=\mathrm{B}_{1 \mathrm{u}}+\mathrm{B}_{2 \mathrm{u}}+\mathrm{B}_{3 \mathrm{u}}\right)$ & $\mathrm{WP} 2=[4 \mathrm{c}, 8 \mathrm{~d}]$ & $\begin{array}{l}\mathrm{B}_{1 \mathrm{u}}=2 \\
\mathrm{~B}_{2 \mathrm{u}}=2 \\
\mathrm{~B}_{3 \mathrm{u}}=2\end{array}$ & $\begin{array}{l}\mathrm{A}_{\mathrm{g}}=2 \\
\mathrm{~B}_{1 \mathrm{~g}}=2 \\
\mathrm{~B}_{2 \mathrm{~g}}=2 \\
\mathrm{~B}_{3 \mathrm{~g}}=2\end{array}$ & $\begin{array}{l}\mathrm{A}_{\mathrm{u}}=2 \\
\mathrm{~B}_{1 \mathrm{u}}=2 \\
\mathrm{~B}_{2 \mathrm{u}}=2 \\
\mathrm{~B}_{3 \mathrm{u}}=2\end{array}$ \\
\hline $\begin{array}{c}\mathrm{HgCl} 18,9,10 \& \\
\mathrm{HgBr} 1\end{array}$ & $\left(\Gamma_{\text {acoustic }}=\mathrm{A}_{\mathrm{u}}+2 \mathrm{~B}_{\mathrm{u}}\right)$ & $\mathrm{WP} 1=[4 \mathrm{e}]$ & $\begin{array}{l}\mathrm{A}_{\mathrm{u}}=1 \\
\mathrm{~B}_{\mathrm{u}}=1\end{array}$ & $\begin{array}{l}A_{g}=1 \\
B_{g}=1\end{array}$ & $\begin{array}{l}\mathrm{A}_{\mathrm{u}}=1 \\
\mathrm{~B}_{\mathrm{u}}=1\end{array}$ \\
\hline $\mathrm{HgBr} 2$ & $\left(\Gamma_{\text {acoustic }}=A_{1}+B_{1}+B_{2}\right)$ & $\mathrm{WP} 1=[4 \mathrm{a}]$ & $\begin{array}{l}\mathrm{A}_{1}=1 \\
\mathrm{~B}_{1}=1 \\
\mathrm{~B}_{2}=1\end{array}$ & $\begin{array}{l}\mathrm{A}_{1}=1 \\
\mathrm{~A}_{2}=1 \\
\mathrm{~B}_{1}=1 \\
\mathrm{~B}_{2}=1\end{array}$ & $\begin{array}{l}\mathrm{A}_{1}=1 \\
\mathrm{~B}_{1}=1 \\
\mathrm{~B}_{2}=1\end{array}$ \\
\hline $\mathrm{HgBr} 3,4$ & $\left(\Gamma_{\text {acoustic }}=A_{u}+2 B_{u}\right)$ & $\mathrm{WP} 1=[4 \mathrm{e}]$ & $\begin{array}{l}\mathrm{A}_{\mathrm{u}}=1 \\
\mathrm{~B}_{\mathrm{u}}=1\end{array}$ & $\begin{array}{l}A_{g}=1 \\
B_{g}=1\end{array}$ & $\begin{array}{l}A_{u}=1 \\
B_{u}=1\end{array}$ \\
\hline $\mathrm{HgBr} 5$ & $\left(\Gamma_{\text {acoustic }}=\mathrm{A}_{\mathrm{u}}+{ }^{1} \mathrm{E}_{\mathrm{u}}+{ }^{2} \mathrm{E}_{\mathrm{u}}\right)$ & $\begin{array}{c}\text { WP6 }=[2 \mathrm{c}, 2 \mathrm{e}, 2 \mathrm{f}, 4 \mathrm{i}, \\
4 \mathrm{j}, 8 \mathrm{k}]\end{array}$ & $\begin{aligned} \mathrm{A}_{\mathrm{u}} & =6 \\
{ }^{1} \mathrm{E}_{\mathrm{u}} & =6 \\
{ }^{2} \mathrm{E}_{\mathrm{u}} & =6\end{aligned}$ & $\begin{aligned} \mathrm{A}_{\mathrm{g}} & =3 \\
\mathrm{~B}_{\mathrm{g}} & =5 \\
{ }^{1} \mathrm{E}_{\mathrm{g}} & =5 \\
{ }^{2} \mathrm{E}_{\mathrm{g}} & =5\end{aligned}$ & $\begin{aligned} \mathrm{A}_{\mathrm{u}} & =6 \\
\mathrm{~B}_{\mathrm{u}} & =4 \\
{ }^{1} \mathrm{E}_{\mathrm{u}} & =6 \\
{ }^{2} \mathrm{E}_{\mathrm{u}} & =6\end{aligned}$ \\
\hline $\mathrm{HgBr6}$ & $\left(\Gamma_{\text {acoustic }}=A_{u}+2 B_{u}\right)$ & WP1 $=[8 \mathrm{f}]$ & $\begin{array}{l}\mathrm{A}_{\mathrm{u}}=1 \\
\mathrm{~B}_{\mathrm{u}}=1\end{array}$ & $\begin{array}{l}\mathrm{A}_{\mathrm{g}}=1 \\
\mathrm{~B}_{\mathrm{g}}=1\end{array}$ & $\begin{array}{l}\mathrm{A}_{\mathrm{u}}=1 \\
\mathrm{~B}_{\mathrm{u}}=1\end{array}$ \\
\hline $\mathrm{HgBr} 7$ & $\left(\Gamma_{\text {acoustic }}=\mathrm{B}_{1 \mathrm{u}}+\mathrm{B}_{2 \mathrm{u}}+\mathrm{B}_{3 \mathrm{u}}\right)$ & $\mathrm{WP} 3=[16 \mathrm{~g}, 32 \mathrm{~h}, 8 \mathrm{a}]$ & $\begin{array}{l}\mathrm{B}_{1 \mathrm{u}}=3 \\
\mathrm{~B}_{2 \mathrm{u}}=3 \\
\mathrm{~B}_{3 \mathrm{u}}=3\end{array}$ & $\begin{array}{l}\mathrm{A}_{\mathrm{g}}=2 \\
\mathrm{~B}_{1 \mathrm{~g}}=3 \\
\mathrm{~B}_{2 \mathrm{~g}}=3 \\
\mathrm{~B}_{3 \mathrm{~g}}=3\end{array}$ & $\begin{array}{l}\mathrm{A}_{\mathrm{u}}=2 \\
\mathrm{~B}_{1 \mathrm{u}}=3 \\
\mathrm{~B}_{2 \mathrm{u}}=3 \\
\mathrm{~B}_{3 \mathrm{u}}=3\end{array}$ \\
\hline $\mathrm{HgBr} 8$ & $\left(\Gamma_{\text {acoustic }}=A_{u}+2 B_{u}\right)$ & $\mathrm{WP} 1=[8 \mathrm{f}]$ & $\begin{array}{l}\mathrm{A}_{\mathrm{u}}=1 \\
\mathrm{~B}_{\mathrm{u}}=1\end{array}$ & $\begin{array}{l}A_{g}=1 \\
B_{g}=1\end{array}$ & $\begin{array}{l}A_{u}=1 \\
B_{u}=1\end{array}$ \\
\hline $\mathrm{HgBr} 9$ & $\left(\Gamma_{\text {acoustic }}=3 \mathrm{~A}_{\mathrm{u}}\right)$ & $\mathrm{WP} 1=[2 \mathrm{i}]$ & $\mathrm{A}_{\mathrm{u}}=1$ & $\mathrm{~A}_{\mathrm{g}}=1$ & $\mathrm{~A}_{\mathrm{u}}=1$ \\
\hline $\mathrm{HgBr} 10$ & $\left(\Gamma_{\text {acoustic }}=A_{u}+2 B_{u}\right)$ & WP1 = [8f $]$ & $\begin{array}{l}\mathrm{A}_{\mathrm{u}}=1 \\
\mathrm{~B}_{\mathrm{u}}=1\end{array}$ & $\begin{array}{l}A_{g}=1 \\
B_{g}=1\end{array}$ & $\begin{array}{l}\mathrm{A}_{\mathrm{u}}=1 \\
\mathrm{~B}_{\mathrm{u}}=1\end{array}$ \\
\hline $\mathrm{HgBr} 11$ & $\left(\Gamma_{\text {acoustic }}=A_{u}+2 B_{u}\right)$ & $\mathrm{WP} 1=[4 \mathrm{e}]$ & $\begin{array}{l}\mathrm{A}_{\mathrm{u}}=1 \\
\mathrm{~B}_{\mathrm{u}}=1\end{array}$ & $\begin{array}{l}\mathrm{A}_{\mathrm{g}}=1 \\
\mathrm{~B}_{\mathrm{g}}=1\end{array}$ & $\begin{array}{l}\mathrm{A}_{\mathrm{u}}=1 \\
\mathrm{~B}_{\mathrm{u}}=1\end{array}$ \\
\hline $\mathrm{HgBr} 12$ & $\left(\Gamma_{\text {acoustic }}=A_{\mathrm{u}}+2 \mathrm{~B}_{\mathrm{u}}\right)$ & $\mathrm{WP} 2=[4 \mathrm{e}, 8 \mathrm{f}]$ & $\begin{array}{l}\mathrm{A}_{\mathrm{u}}=2 \\
\mathrm{~B}_{\mathrm{u}}=2\end{array}$ & $\begin{array}{l}A_{g}^{g}=2 \\
B_{g}=2\end{array}$ & $\begin{array}{l}\mathrm{A}_{\mathrm{u}}=2 \\
\mathrm{~B}_{\mathrm{u}}=2\end{array}$ \\
\hline $\mathrm{HgBr} 13$ & $\left(\Gamma_{\text {acoustic }}=\mathrm{B}_{1 \mathrm{u}}+\mathrm{B}_{2 \mathrm{u}}+\mathrm{B}_{3 \mathrm{u}}\right)$ & $\mathrm{WP} 2=[4 \mathrm{~d}, 8 \mathrm{e}]$ & $\begin{array}{l}\mathrm{B}_{1 \mathrm{u}}=2 \\
\mathrm{~B}_{2 \mathrm{u}}=2 \\
\mathrm{~B}_{3 \mathrm{u}}=2\end{array}$ & $\begin{array}{l}\mathrm{A}_{\mathrm{g}}=2 \\
\mathrm{~B}_{1 \mathrm{~g}}=2 \\
\mathrm{~B}_{2 \mathrm{~g}}=2 \\
\mathrm{~B}_{3 \mathrm{~g}}=2\end{array}$ & $\begin{array}{l}\mathrm{A}_{\mathrm{u}}=2 \\
\mathrm{~B}_{1 \mathrm{u}}=2 \\
\mathrm{~B}_{2 \mathrm{u}}=2 \\
\mathrm{~B}_{3 \mathrm{u}}=2\end{array}$ \\
\hline $\mathrm{HgBr} 14$ & $\left(\Gamma_{\text {acoustic }}=A_{u}+2 B_{u}\right)$ & $\mathrm{WP} 1=[4 \mathrm{e}]$ & $\begin{array}{l}\mathrm{A}_{\mathrm{u}}=1 \\
\mathrm{~B}_{\mathrm{u}}=1\end{array}$ & $\begin{array}{l}A_{g}=1 \\
B_{g}=1\end{array}$ & $\begin{array}{l}\mathrm{A}_{\mathrm{u}}=1 \\
\mathrm{~B}_{\mathrm{u}}=1\end{array}$ \\
\hline $\operatorname{HgBr} 15,16,17$ & $\left(\Gamma_{\text {acoustic }}=3 \mathrm{~A}_{\mathrm{u}}\right)$ & $\mathrm{WP} 1=[2 \mathrm{i}]$ & $\mathrm{A}_{\mathrm{u}}=1$ & $A_{g}=1$ & $A_{u}=1$ \\
\hline
\end{tabular}




\begin{tabular}{|c|c|c|c|c|c|}
\hline $\mathrm{HgBr} 18$ & $\left(\Gamma_{\text {acoustic }}=\mathrm{A}_{\mathrm{u}}+2 \mathrm{~B}_{\mathrm{u}}\right)$ & $\mathrm{WP} 2=[4 \mathrm{e}, 8 \mathrm{f}]$ & $\begin{array}{l}\mathrm{A}_{\mathrm{u}}=2 \\
\mathrm{~B}_{\mathrm{u}}=2\end{array}$ & $\begin{array}{l}\mathrm{A}_{\mathrm{g}}=2 \\
\mathrm{~B}_{\mathrm{g}}=2\end{array}$ & $\begin{array}{l}\mathrm{A}_{\mathrm{u}}=2 \\
\mathrm{~B}_{\mathrm{u}}=2\end{array}$ \\
\hline HgI1 & $\left(\Gamma_{\text {acoustic }}=3 \mathrm{~A}_{\mathrm{u}}\right)$ & $\mathrm{WP} 1=[2 \mathrm{i}]$ & $\mathrm{A}_{\mathrm{u}}=1$ & $A_{g}=1$ & $\mathrm{~A}_{\mathrm{u}}=1$ \\
\hline $\mathrm{HgI} 2$ & $\left(\Gamma_{\text {acoustic }}=\mathrm{A}_{\mathrm{u}}+{ }^{1} \mathrm{E}_{\mathrm{u}}+{ }^{2} \mathrm{E}_{\mathrm{u}}\right)$ & $\mathrm{WP} 3=[16 \mathrm{f}, 4 \mathrm{a}, 4 \mathrm{~b}]$ & $\begin{aligned} \mathrm{A}_{\mathrm{u}} & =3 \\
{ }^{1} \mathrm{E}_{\mathrm{u}} & =3 \\
{ }^{2} \mathrm{E}_{\mathrm{u}} & =3\end{aligned}$ & $\begin{aligned} \mathrm{A}_{\mathrm{g}} & =1 \\
\mathrm{~B}_{\mathrm{g}} & =3 \\
{ }^{1} \mathrm{E}_{\mathrm{g}} & =3 \\
{ }^{2} \mathrm{E}_{\mathrm{g}} & =3\end{aligned}$ & $\begin{aligned} \mathrm{A}_{\mathrm{u}} & =3 \\
\mathrm{~B}_{\mathrm{u}} & =1 \\
{ }^{1} \mathrm{E}_{\mathrm{u}} & =3 \\
{ }^{2} \mathrm{E}_{\mathrm{u}} & =3\end{aligned}$ \\
\hline $\mathrm{HgI3}$ & $\left(\Gamma_{\text {acoustic }}=3 \mathrm{~A}_{\mathrm{u}}\right)$ & $\mathrm{WP} 2=[1 \mathrm{f}, 2 \mathrm{i}]$ & $A_{u}=2$ & $A_{g}=1$ & $\mathrm{~A}_{\mathrm{u}}=2$ \\
\hline $\mathrm{HgI} 4$ & $\left(\Gamma_{\text {acoustic }}=A_{u}+2 B_{u}\right)$ & $\mathrm{WP} 2=[4 \mathrm{e}, 8 \mathrm{f}]$ & $\begin{array}{l}\mathrm{A}_{\mathrm{u}}=2 \\
\mathrm{~B}_{\mathrm{u}}=2\end{array}$ & $\begin{array}{l}\mathrm{A}_{\mathrm{g}}=2 \\
\mathrm{~B}_{\mathrm{g}}=2\end{array}$ & $\begin{array}{l}A_{u}=2 \\
B_{u}=2\end{array}$ \\
\hline $\mathrm{HgI5}$ & $\left(\Gamma_{\text {acoustic }}=A_{1}+B_{1}+B_{2}\right)$ & $\mathrm{WP} 1=[4 \mathrm{a}]$ & $\begin{array}{l}\mathrm{A}_{1}=1 \\
\mathrm{~B}_{1}=1 \\
\mathrm{~B}_{2}=1\end{array}$ & $\begin{array}{l}\mathrm{A}_{1}=1 \\
\mathrm{~A}_{2}=1 \\
\mathrm{~B}_{1}=1 \\
\mathrm{~B}_{2}=1\end{array}$ & $\begin{array}{l}\mathrm{A}_{1}=1 \\
\mathrm{~A}_{2}=1 \\
\mathrm{~B}_{1}=1 \\
\mathrm{~B}_{2}=1\end{array}$ \\
\hline HgI6 & $\left(\Gamma_{\text {acoustic }}=B_{1 u}+B_{2 u}+B_{3 u}\right)$ & $\mathrm{WP} 2=[4 \mathrm{c}, 8 \mathrm{~d}]$ & $\begin{array}{l}\mathrm{B}_{1 \mathrm{u}}=2 \\
\mathrm{~B}_{2 \mathrm{u}}=2 \\
\mathrm{~B}_{3 \mathrm{u}}=2\end{array}$ & $\begin{array}{l}\mathrm{A}_{\mathrm{g}}=1 \\
\mathrm{~B}_{1 \mathrm{~g}}=2 \\
\mathrm{~B}_{2 \mathrm{~g}}=2 \\
\mathrm{~B}_{3 \mathrm{~g}}=2\end{array}$ & $\begin{array}{l}\mathrm{A}_{\mathrm{u}}=2 \\
\mathrm{~B}_{1 \mathrm{u}}=2 \\
\mathrm{~B}_{2 \mathrm{u}}=2 \\
\mathrm{~B}_{3 \mathrm{u}}=2\end{array}$ \\
\hline $\mathrm{HgI7}$ & $\left(\Gamma_{\text {acoustic }}=A_{2 u}+E_{u}\right)$ & $\mathrm{WP} 3=[16 \mathrm{~d}, 16 \mathrm{e}, 32 \mathrm{~g}]$ & $\begin{aligned} \mathrm{A}_{2 \mathrm{u}} & =3 \\
\mathrm{E}_{\mathrm{u}} & =3\end{aligned}$ & $\begin{array}{c}A_{1 g}=3 \\
B_{1 g}=3 \\
B_{2 g}=3 \\
E_{g}=3\end{array}$ & $\begin{aligned} \mathrm{A}_{1 \mathrm{u}} & =3 \\
\mathrm{~A}_{2 \mathrm{u}} & =3 \\
\mathrm{~B}_{1 \mathrm{u}} & =3 \\
\mathrm{~B}_{2 \mathrm{u}} & =3 \\
\mathrm{E}_{\mathrm{u}} & =3\end{aligned}$ \\
\hline HgI8 & $\left(\Gamma_{\text {acoustic }}=A_{2 u}+E_{u}\right)$ & $\mathrm{WP} 3=[16 \mathrm{~g}, 16 \mathrm{~h}, 8 \mathrm{e}]$ & $\begin{array}{c}\mathrm{A}_{2 \mathrm{u}}=3 \\
\mathrm{E}_{\mathrm{u}}=3\end{array}$ & $\begin{array}{c}\mathrm{A}_{1 \mathrm{~g}}=3 \\
\mathrm{~B}_{1 \mathrm{~g}}=3 \\
\mathrm{~B}_{2 \mathrm{~g}}=3 \\
\mathrm{E}_{\mathrm{g}}=3\end{array}$ & $\begin{aligned} \mathrm{A}_{1 \mathrm{u}} & =2 \\
\mathrm{~A}_{2 \mathrm{u}} & =3 \\
\mathrm{~B}_{1 \mathrm{u}} & =2 \\
\mathrm{~B}_{2 \mathrm{u}} & =3 \\
\mathrm{E}_{\mathrm{u}} & =3\end{aligned}$ \\
\hline $\mathrm{HgI9}$ & $\left(\Gamma_{\text {acoustic }}=A_{2 \mathrm{u}}+\mathrm{E}_{\mathrm{u}}\right)$ & $\mathrm{WP} 4=[4 \mathrm{c}, 4 \mathrm{~d}, 8 \mathrm{f}, 8 \mathrm{~g}]$ & $\begin{aligned} \mathrm{A}_{2 \mathrm{u}} & =4 \\
\mathrm{E}_{\mathrm{u}} & =4\end{aligned}$ & $\begin{array}{c}\mathrm{A}_{1 \mathrm{~g}}=4 \\
\mathrm{~B}_{1 \mathrm{~g}}=4 \\
\mathrm{~B}_{2 \mathrm{~g}}=2 \\
\mathrm{E}_{\mathrm{g}}=4\end{array}$ & $\begin{aligned} \mathrm{A}_{1 \mathrm{u}} & =2 \\
\mathrm{~A}_{2 \mathrm{u}} & =4 \\
\mathrm{~B}_{1 \mathrm{u}} & =2 \\
\mathrm{~B}_{2 \mathrm{u}} & =4 \\
\mathrm{E}_{\mathrm{u}} & =4\end{aligned}$ \\
\hline HgI10 & $\left(\Gamma_{\text {acoustic }}=3 \mathrm{~A}_{\mathrm{u}}\right)$ & $\mathrm{WP} 1=[2 \mathrm{i}]$ & $\mathrm{A}_{\mathrm{u}}=1$ & $A_{g}=1$ & $\mathrm{~A}_{\mathrm{u}}=1$ \\
\hline HgI11 & $\left(\Gamma_{\text {acoustic }}=\mathrm{A}_{\mathrm{u}}+2 \mathrm{~B}_{\mathrm{u}}\right)$ & $\mathrm{WP} 2=[4 \mathrm{e}, 8 \mathrm{f}]$ & $\begin{array}{l}A_{u}=2 \\
B_{u}=2\end{array}$ & $\begin{array}{l}A_{g}=2 \\
B_{g}=2\end{array}$ & $\begin{array}{l}A_{u}=2 \\
B_{u}=2\end{array}$ \\
\hline HgI12,13 & $\left(\Gamma_{\text {acoustic }}=\mathrm{A}_{\mathrm{u}}+2 \mathrm{~B}_{\mathrm{u}}\right)$ & $\mathrm{WP} 1=[4 \mathrm{e}]$ & $\begin{array}{l}\mathrm{A}_{\mathrm{u}}=1 \\
\mathrm{~B}_{\mathrm{u}}=1\end{array}$ & $\begin{array}{l}\mathrm{A}_{\mathrm{g}}=1 \\
\mathrm{~B}_{\mathrm{g}}=1\end{array}$ & $\begin{array}{l}\mathrm{A}_{\mathrm{u}}=1 \\
\mathrm{~B}_{\mathrm{u}}=1\end{array}$ \\
\hline HgI14 & $\left(\Gamma_{\text {acoustic }}=\mathrm{A}_{\mathrm{u}}+2 \mathrm{~B}_{\mathrm{u}}\right)$ & $\mathrm{WP} 2=[4 \mathrm{e}, 8 \mathrm{f}]$ & $\begin{array}{l}\mathrm{A}_{\mathrm{u}}=2 \\
\mathrm{~B}_{\mathrm{u}}=2\end{array}$ & $\begin{array}{l}\mathrm{A}_{\mathrm{g}}=2 \\
\mathrm{~B}_{\mathrm{g}}=2\end{array}$ & $\begin{array}{l}\mathrm{A}_{\mathrm{u}}=2 \\
\mathrm{~B}_{\mathrm{u}}=2\end{array}$ \\
\hline HgI15 & $\left(\Gamma_{\text {acoustic }}=A_{\mathrm{u}}+2 \mathrm{~B}_{\mathrm{u}}\right)$ & WP1 $=[8 \mathrm{f}]$ & $\begin{array}{l}\mathrm{A}_{\mathrm{u}}=1 \\
\mathrm{~B}_{\mathrm{u}}=1\end{array}$ & $\begin{array}{l}A_{g}=1 \\
B_{g}=1\end{array}$ & $\begin{array}{l}\mathrm{A}_{\mathrm{u}}=1 \\
\mathrm{~B}_{\mathrm{u}}=1\end{array}$ \\
\hline HgI16 & $\left(\Gamma_{\text {acoustic }}=3 \mathrm{~A}_{\mathrm{u}}\right)$ & $\mathrm{WP} 1=[2 \mathrm{i}]$ & $A_{u}=1$ & $A_{g}=1$ & $A_{u}=1$ \\
\hline HgI17 & $\left(\Gamma_{\text {acoustic }}=\mathrm{A}_{\mathrm{u}}+2 \mathrm{~B}_{\mathrm{u}}\right)$ & $\mathrm{WP} 1=[4 \mathrm{e}]$ & $\begin{array}{l}A_{u}=1 \\
B_{u}=1\end{array}$ & $\begin{array}{l}\mathrm{A}_{\mathrm{g}}=1 \\
\mathrm{~B}_{\mathrm{g}}=1\end{array}$ & $\begin{array}{l}\mathrm{A}_{\mathrm{u}}=1 \\
\mathrm{~B}_{\mathrm{u}}=1\end{array}$ \\
\hline HgI18 & $\left(\Gamma_{\text {acoustic }}=2 \mathrm{~A}^{\prime}+\mathrm{A}^{\prime \prime}\right)$ & $\mathrm{WP} 1=[4 \mathrm{a}]$ & $\begin{array}{l}\mathrm{A}^{\prime}=1 \\
\mathrm{~A}^{\prime}=1\end{array}$ & $\begin{array}{l}\mathrm{A}^{\prime}=1 \\
\mathrm{~A}^{\prime}=1\end{array}$ & $\begin{array}{l}\mathrm{A}^{\prime}=1 \\
\mathrm{~A}^{\prime \prime}=1\end{array}$ \\
\hline
\end{tabular}




\section{Conclusion}

Due to the unique chemical nature of halogen bonding, this intermolecular interaction serves as an additional tool for the development of crystal engineering. The nature of intermolecular interactions between halogen atoms, $\mathrm{X} \cdots \mathrm{X}(\mathrm{X}=\mathrm{Cl}, \mathrm{Br}, \mathrm{I})$, continues to be of topical interest because these interactions may be used as design elements in crystal engineering. Interactions between halogen atoms such as $\mathrm{Cl} \cdots \mathrm{Cl}, \mathrm{Br} \cdots \mathrm{Br}$ and $\mathrm{I} \cdots \mathrm{I}$ are special cases of halogen bonding. It has become obviously clear that halogen...halogen interaction could very well be utilized as a design element in crystal engineering. On the basis of the short distances obtained between X---X, the halogen interactions are undoubtedly the vital forces responsible for stabilizing a crystal and definitely can play a pivoting role along with $\mathrm{M}---\mathrm{M}$ and other non-covalent interactions in a crystal structure.

\section{References}

1. Desiraju G R, Crystal Engineering: The design of organic solids (Amsterdam, Elsevier, 1987.

2. Desiraju G R, Crystal Engineering, Structure and function Perspectives in supramolecular chemistry (Chichester, Wiley, 1989.

3. Tiekink E R and Vittal J J, Frontiers in crystal engineering (Chichester, Wiley, 2005).

4. Braga D, Grepioni F and Orpan A G, Crystal Engineering: From molecules to crystals to materials (Dordrecht, Kluwer, 1999.

5. Aakeroy C B, Acta Crystallogr., Sect B: 1997, 53, 569-586; DOI:10.1107/S0108768197008446

6. Goldberg I, Chem Commun., 2005, 1243-1254; DOI:10.1039/B416425C

7. Bishop R, Synlett., 1999, 1351-1358; DOI:10.1055/s-1999-2833

8. Biradha K, Cryst Eng Comm., 2003, 5, 374-384; DOI:10.1039/B309903B

9. Aakeroy C B, Champness N R and Janiak C, Cryst Eng Comm., 2010, 12, 22-43; DOI:10.1039/B919819A

10. Dastidar P, Chem Soc Ev., 2008, 37, 2699-2715; DOI:10.1039/B807346E

11. Glaser R, Acc Chem Res., 2007, 40(1), 9-17; DOI:10.1021/ar0301633

12. Braga D and Grepioni F, Chem Commun., 2005, 3635-3645; DOI:10.1039/B504668H

13. Braga D, Brammer L and Champness N R, Cryst Eng Comm., 2005, 7, 1-19; DOI:10.1039/B417413E

14. Erk P, Hengelsberg H, Haddow M F and van Gelder R,, Cryst Eng Comm., 2004, 6, 474-483; DOI:10.1039/b409282a

15. Sharma C V K, Cryst Growth Des., 2002, 2(6), 465-474; DOI:10.1021/cg0200356

16. Desiraju G R, Crystal Engg., A brief Overview., 2010, 122(5), 667-675.

17. Chujo Y, Organic-Inorganic Nano-Hybrid Materials, (konaNo. 25, 2007), 255.

18. Coronado E, Clemente-Leon M, Galan-Mascaros J R, Gimenez-Saiz C, GomezGarcia C J and Martinez-Ferrero E, J Chem Soc., Dalton Trans,. 2000, 3955-3961.

19. Albright T A, Hoffmann P and Hoffmann R, J Am Chem Soc., 1987, 109, 3240.

20. Desiraju G R and Parthasarathi R, J Am Chem Soc., 1989, 111(23), 8725-8726; DOI:10.1021/ja00205a027

21. Price S L, Stone A J, Lucas J, Rowland R S, Thornley A E, J Am Chem Soc., 1994, 116(11), 4910-4918; DOI:10.1021/ja00090a041

22. (a) Desiraju G R, in Organic Solid State Chemistry, (Elsevier, Amsterdam, 1987); (b) Navon O, Bernstein J and Khodorkovsky V, Angew Chem Int Ed Engl., 1997, 36(6), 601-603; DOI:10.1002/anie.199706011 (c) Bosch E and Barnes C L, Cryst Growth Des., 2002, 2(4), 299-302; DOI:10.1021/cg025517w (d) Nangia A, Cryst Eng 
Comm., 2002, 4, 93-101; DOI:10.1039/B201206E, (e) Saha B K, Jetti R K R, Reddy L S, Aitipamula S and Nangia A, Cryst Growth Des., 2005, 5(3), 887-899; DOI:10.1021/cg049691r (f) Paulini R, Muller K and Diederich F, Angew Chem Int Ed Engl., 2005, 44(12), 1788-1805; DOI:10.1002/anie.200462213 (g) Zordan F, Brammer L and Sherwood P, J Am Chem Soc., 2005, 127(16), 5979-5989; DOI:10.1021/ja0435182

23. Brandenburg K and Putz H and Diamond, Crystal Impact GbR, Bonn, Germany, 1999.

24. Ponnuswamy M N and Trotter, Acta Crystallogr Sect C, 1984, 40, 1671-1673; DOI:10.1107/S0108270184009112

25. Aharoni A, Kapon M and Reisner G M, Acta Crystallogr Sect C, 1989, 45, 40-44; DOI:10.1107/S0108270188010248

26. Zouari F, BenSalah A and Hovestreydt E R, Acta Crystallogr Sect C, 1995, 51, 1563 1565; DOI:10.1107/S0108270194013454

27. Spengler R, Zouari F, BenSalah A, Burzlaff H, Acta Crystallogr Sect C, 1997, 53, 1407-1409; DOI:10.1107/S0108270197008512

28. Spengler R, Zouari F, BenSalah A and Burzlaff H, Acta Crystallogr Sect C., 1997, 53, 1566-1568; DOI:10.1107/S0108270197008500

29. Spengler R, Zouari R, Zimmermann H, BenSalah A and Burzlaff H, Acta Crystallogr Sect C, 1998, 54, 1628-1631; DOI:10.1107/S0108270198008282

30. Spengler R, Zouari R, Zimmermann H, BenSalah A and Burzlaff H, Acta Crystallogr Sect C, 1998, 54, ICU9800034, DOI:10.1107/S0108270198099491

31. Amami M, Smaolen S and Bensalah A, Acta Crystallogr Sect E, 2002, 58, m416m419; DOI:10.1107/S160053680201214X

32. Muir K W, Morris D G and Chii C O, Acta Crystallogr Sect E, 2004, 60, m60, DOI:10.1107/S0108270103028464

33. Florke U, Herres-Pawlis S, Heuwing A, Neuba A, Seewald O and Henkel G, Acta Crystallogr Sect C, 2006, 62, m234-m237; DOI:10.1107/S0108270106014429

34. Thiel J, Bu X and Coppens P, Acta Crystallogr Sect C., 1994, 50, 23-25; DOI:10.1107/S0108270193006912

35. Lutz M, Spek A L, Markies P R, Akkerman O S and Bickelhaupt F, Acta Crystallogr Sect C, 1998, 54, 1091-1093; DOI:10.1107/S0108270198002418

36. Matcoviv-Calogovic D, Popovic J, Popovic Z, Picek I, Soldin Z, Acta Crystallogr Sect C, 2002, 58, m39-m40; http://dx.doi.org/10.1107/S0108270101018613

37. Nockemann P and Meyer G, Acta Crystallogr Sect E, 2002, 58, m529-m530; DOI:10.1107/S1600536802015337

38. Nockemann P and Meyer G, Acta Crystallogr Sect E, 2002, 58, m531-533; DOI:10.1107/S1600536802015301

39. Popovic Z, Pavlovic G, Matkovic-Calogovic D and Soldin Z, Acta Crystallogr Sect C, 2003, 59, m165-167; DOI:10.1107/S0108270103005705

40. Matkovic-Calogovic D, Mrvos-Sermek D, Popovic Z and Soldin Z, Acta Crystallogr Sect C, 2004, 60, m44-m46; DOI:10.1107/S0108270103028014

41. Liu C B, Che G B, Cui Y C and Li C B, Acta Crystallogr Sect E, 2005, 61, m2469m2470; DOI:10.1107/S1600536805035348

42. Che G B, Liu C B, Cui Y C and Li C B, Acta Crystallogr Sect E, 2005, 61, m2704m2705; DOI:10.1107/S1600536805038754

43. Gong J B, Zhou L N and Chen W, Acta Crystallogr Sect E, 2006, 62, m440-m441; DOI:10.1107/S1600536806002728 
44. Sabounchei S J, Dadras A, Jodaian V, Nemattalab H and Khavasi H R, Acta Crystallogr Sect E, 2008, 64, m833; DOI:10.1107/S1600536808001220

45. Terao H, Gesing T M, Ishihara H, Furukawa Y and Gowda B T, Acta Crystallogr Sect E, 2009, 65, m323; DOI:10.1107/S1600536809005972

46. Al-Far R H, Haddad S F and Ali B F, Acta Crystallogr Sect C, 2009, 65, m451-454; DOI:10.1107/S010827010903772X

47. Rofouei M K, Beiza A and Attar Gharamaleki J, Acta Crystallogr Sect E, 2009, 65, m1259-m1260; DOI:10.1107/S1600536809038732

48. Yang R, Ma J P, Huang R Q and Dong Y B, Acta Crystallogr Sect C, 2011, 67, m176-m180; DOI:10.1107/S0108270111016246

49. Abedi A, Acta Crystallogr Sect E, 2011, 67, m76-m77;

DOI:10.1107/S1600536810051494

50. Ghiasi G, Acta Crystallogr Sect E, 2011, 67, m595; DOI:10.1107/S1600536811013274

51. Zhang W S, Liu Z J, Xu F and Xun Q N, Acta Crystallogr Sect E, 2011, 67, m1849; DOI:10.1107/S1600536811049993

52. $\mathrm{Bu} \mathrm{X}$ and Coppens $\mathrm{P}$, Acta Crystallogr Sect C., 1992, 48, 1565-1566; DOI:10.1107/S0108270192005298

53. Bengtsson-kloo L, Berglund J, Stegemann H, Svensson C and Svensson P H, Acta Crystallogr Sect C, 1998, 54, 8151, DOI:10.1107/S0108270198008889

54. $\mathrm{Hu}$ N H, Liu Y S and Aoki K, Acta Crystallogr Sect C, 1999, 55, 304-308; DOI:10.1107/S0108270198013407

55. Pavlovic G, Popovic Z, Soldin Z, Matkovic-Calogovic D, Acta Crystallogr Sect C, 2003, 56, 801-803.

56. Matkovic-Calogovic D, Popovic Z, Pavlovic G, Soldin Z and Giester G, Acta Crystallogr Sect C, 2001, 57, 409-411; DOI:10.1107/S0108270101001822

57. Hostettler M and Schwarzenbach D, Acta Crystallogr Sect B, 2002, 58, 914-920; DOI: $10.1107 /$ S0108768102016191

58. Hostettler M, Birkedal H and Schwarzenbach D, Acta Crystallogr Sect B, 2003, 58, 903-913; DOI:10.1107/S010876810201618X

59. Nockemann P and Meyer G, Acta Crystallogr Sect E., 2003, 59, m236; DOI: $10.1107 /$ S1600536803007335

60. Yusof M S M, Yamin B M and Kassim M B, Acta Crystallogr Sect E., 2004, 60, m98-m99; DOI:10.1107/S1600536803028654

61. Chen J X, Zhang W H, Ren Z G, Zhang Y and Lang J P, Acta Crystallogr Sect E, 2005, 61, m60-m62; DOI:10.1107/S1600536804031630

62. Oh M J, Lee Y M, Lee S J, Kang S K and Choi S N, Acta Crystallogr Sect C, 2006, 62, m51-m53; DOI:10.1107/S0108270105043088

63. Popovic Z, Pavlovic G, Soldin Z, Acta Crystallogr., 2006, Sect. C: 62, m272.

64. Tan X J, Jin L Q and Xing D X, Acta Crystallogr Sect E, 2006, 62, m2502-m2504; DOI: $10.1107 / \mathrm{S} 1600536806035380$

65. Yang R, Ma J P, Huang R Q and Dong Y B, Acta Crystallogr Sect C, 2011, 67, m176-m180; DOI:10.1107/S0108270111016246

66. Li T L, Acta Crystallogr Sect E, 2011, 67, m1396; DOI:10.1107/S1600536811037160

67. Ghaemi A, Shojaiean R, Ng S W and Tiekink E R T, Acta Crystallogr Sect E, 2011, 67, m1449-m1450; DOI:10.1107/S1600536811038748 